\title{
Modelo de programación lineal en el diseño de la red de tuberías y costo del sistema de riego por goteo para determinar la superficie mínima de operación en diferentes cultivos en un suelo salino
}

\author{
Linear programming in the design of pipes with drip irrigation for determining optimal minimum \\ size in crops on saline soils
}

\section{Lía Ramos Fernández ${ }^{1}$}

\section{Resumen}

Las altas inversiones que involucra el uso del riego presurizado lleva a emplear óptimamente los recursos, a fin de maximizar los beneficios y recuperar la inversión en el menor tiempo posible. Para esto, la programación lineal permite optimizar el funcionamiento de la bomba hidráulica en el cabezal de riego con los diámetros económicos de tubería correspondientes, en función de los costos de la tubería y del bombeo. Por ello, el objetivo del presente estudio es determinar la superficie mínima de operación con cultivos de espárrago, vid, tomate, páprika y cebolla, en suelo salino. Se desarrollaron 25 modelos de programación lineal para determinar el costo mínimo del sistema en tamaños de parcela de 50, 20,15, 10 y 5 ha. Finalmente, se realiza la evaluación económica para definir la superficie mínima de operación según el cultivo evaluado. De los resultados del estudio, se obtienen tamaños de parcela mínimos óptimos de 20 ha para espárrago, 50 ha para vid y 5 ha para tomate, cebolla y ají páprika. De los cinco cultivos propuestos, es la cebolla la que presenta la mayor rentabilidad, seguida, en orden decreciente, del tomate, ají páprika, espárrago y vid. Acorde con el análisis de sensibilidad, para una reducción de un $20 \%$ en el rendimiento, se obtiene que la cebolla sigue siendo rentable en todos los tamaños de parcela; en cambio, para el tomate y ají páprika, solo resultaron rentables en tamaños de parcela de 10, 15, 20 y 50 ha. En el caso de los cultivos de espárrago y vid, ninguno resultó rentable.

Palabras clave: programación lineal; optimización; riego por goteo y diseño de tuberías.

\begin{abstract}
Drip irrigation needs high investment and it requires using resources optimally, to maximize profits and recover the on investment in the shortest possible time. For this reason, linear programming optimizes the operation of the hydraulic pump in the irrigation head with economical diameters of pipes, depending on the pipe cost and pumping cost. Thus, the aim of this study is to determine minimal operation surface in crops of asparagus, grapes, tomatoes, paprika and onion in saline soil. Twenty-five linear programming models were developed to determine the minimal cost of the system for parcel sizes of 50, 20, 15, 10 and 5 ha. Finally, the economic evaluation was performed to define the minimum operation area according to the evaluated crop. From the results of the study, we obtain an optimal minimum land size of 20 ha for asparagus, 50 ha for vine and 5 ha for tomato, onion and paprika. The onion has the highest yield followed in decreasing order by tomato, paprika, asparagus and vine. According to sensitivity analysis for a reduction of $20 \%$ in crop yield, we found that the onion is profitable at all land sizes, whereas for tomato and paprika it was profitable only in land sizes of 10, 15, 20 and 50 ha. In the case of asparagus and vine, none was profitable.
\end{abstract}

Keywords: linear programming; optimization; drip irrigation and pipe design.

\section{Introducción}

Las altas inversiones que requiere el uso del riego presurizado nos lleva a emplear óptimamente los recursos, a fin de maximizar los beneficios y recuperar la inversión en el menor tiempo posible. Para esto, la programación lineal permite optimizar el funcionamiento de la bomba hidráulica en el cabezal de riego con los diámetros económicos de tubería correspondientes, en función de los costos de la tubería y del bombeo. Es así que en la selección de los diámetros de una red de tuberías, además 
de considerar los factores hidráulicos tales como las presiones requeridas, caudales y velocidades máximas permitidas, se debe tener en cuenta los factores económicos tales como el costo de oportunidad del capital, periodo de pago del préstamo, costo unitario de energía y horas de bombeo anual, con el objeto de seleccionar los diámetros de tuberías que produzcan el costo mínimo (Ascencios, 1999; Georgiadis, Schilling, Rotstein y Macchietto, 1999; González-Cebollada y Macarulla, 2012; Swamee y Sharma, 2013). Al respecto, la programación lineal permite obtener el mínimo costo de un sistema de riego a través de una función objetivo que incluye los costos de bombeo y de tubería, los cuales se optimizan de acuerdo a las limitaciones específicas. Las variables de decisión que se utilizan, generalmente, son los diámetros de las tuberías, las pérdidas de carga y las longitudes de tubería; como restricciones, se toma en cuenta las longitudes de la tubería y las cargas piezométricas disponibles, con el fin de cubrir las pérdidas por fricción en la tubería (Ascencios, 1999; Georgiadis et al., 1999; González-Cebollada y Macarulla, 2012). La función objetivo del modelo de optimización está representada por $\mathrm{Z}$ con variables de decisión $\mathrm{L}_{\mathrm{ij}} \mathrm{y} \mathrm{Ht}$ : MinimizarZ $=\sum_{i=1}^{N S} \sum_{j=1}^{N D_{i}} C_{i j} L_{i j}+C H t$. Donde: $\mathrm{C}_{\mathrm{ij}}$ es costo unitario anualizado de los tramos de tubería según diámetro $(\$ / \mathrm{m})$; $\mathrm{L}_{\mathrm{ij}}$ es longitud del tramo de tubería según diámetro $(\mathrm{m}) ; \mathrm{C}$ es el costo anual de bombeo del sistema $(\$ / \mathrm{m})$; Ht es la carga dinámica total $(\mathrm{m})$; ND es el número de diámetros comerciales disponibles en el mercado y NS es el número de tramos en la red.

El costo anualizado de la tubería se obtiene del producto del factor de recuperación del capital (FRC) por el costo unitario de la tubería para un periodo de vida útil (n) en años y un interés bancario (Ascencios, 1999; GonzálezCebollada y Macarulla, 2012). Cuando la fuente de energía es eléctrica, el costo anual de bombeo del sistema depende de la carga dinámica total, de la eficiencia de la unidad de bombeo, del número de horas anuales de bombeo y del costo unitario de energía; este costo, matemáticamente, está representado por $\mathrm{C}=$ Coeficiente ${ }^{*} \mathrm{Ht}$, en que el coeficiente depende de la descarga, la carga dinámica total, la eficiencia de la unidad de bombeo, el número de horas anuales de bombeo y el costo unitario de energía.

La restricción hidráulica permite asegurar la presión mínima (Hmin) en los nudos de la red con el fin de cubrir las pérdidas por fricción en la tubería y se define por la expresión: $\quad H t-\sum^{N S} \sum^{N D} J_{i j} L_{i j} \geq H_{\min }$. Donde: $\mathrm{L}_{\mathrm{ij}}$ son los diferentes tramos de recorrirido del flujo para cada nudo; $\mathrm{J}_{\mathrm{ij}}$ es la pérdida de carga por fricción según diámetro a lo largo del recorrido del flujo para cada nudo $(\mathrm{m} / \mathrm{m})$.

La restricción geométrica permite asegurar que para cada tramo de tubería, la suma de las longitudes parciales de tubería, con diámetros seleccionados, debe ser igual a su longitud (Li), esto es: $L i=\sum_{j=1} L i j$. Donde: $L_{\mathrm{i}}$ es la longitud del tramo de tubería i según diámetros seleccionados

(m). La tercera restricción del modelo plantea que las variables de decisión, como la carga dinámica $(\mathrm{Ht})$ y las longitudes de las tuberías $\mathrm{L}_{\mathrm{ij}}$ no pueden ser negativas, esto es: $\mathrm{L}_{i j} \geq 0 ; \mathrm{Ht} \geq 0$. Finalmente, la función objetivo asociada con las restricciones forman el modelo de optimización. El objetivo del presente estudio es determinar la superficie mínima de operación con cultivos de espárrago, vid, tomate, páprika y cebolla; con este fin, se desarrollan modelos de programación lineal para determinar el costo mínimo del sistema en tamaños de parcela de 50, 20, 15, 10 y 5 ha. Finalmente, se realiza la evaluación económica para definir la superficie mínima de operación en los casos evaluados.

\section{Materiales y métodos}

\section{Zona de estudio}

La zona de estudio son parcelas en la Pampa de Villacurí del departamento de Ica, con una altitud de $357 \mathrm{msnm}$, clima templado-cálido y topografía casi plana, con ligeras pendientes. El suelo es de textura arenosa, con capas duras de sales superficiales de hasta $0,60 \mathrm{~m}$, pero, sometidas a lavado, son cultivadas con éxito. La fuente de agua es la subterránea del acuífero de Ica con buena calidad para el riego ( $\mathrm{C} 2 \mathrm{~S} 1)$, no existen problemas, sin embargo, con la salinidad efectiva y el índice de salinidad potencial debido a que el riego es aplicado con alta frecuencia; es decir, no hay posibilidad que ocurra precipitación de sales solubles, por límite de solubilidad, ni disminución en más del 50 $\%$ de la humedad aprovechable del suelo. Además, el contenido de cloruros y boro está por debajo de los valores de tolerancia de los cultivos sensibles. Esta información actualizada de la zona, además de datos meteorológicos y de cultivos, fue proporcionada por SENAMHI Ica, el Programa Subtectorial de Irrigaciones (PSI Ica) y la Agencia Agraria Ica.

\section{Metodología}

Se realizó la visita de reconocimiento del campo para identificar las parcelas de la zona de estudio, la pendiente, la fuente y calidad del agua, fuente de energía, cultivos y variedades que se adaptaran a las condiciones climáticas y del suelo así como para recopilar información existente de la zona.

Los cultivos considerados fueron espárrago, vid, tomate, páprika y cebolla que se evaluaron para tamaños de parcela de 5, 10, 15, 20 y 50 ha. Por lo que se tendrían 25 combinaciones, producto de los cinco tamaños de parcela por los cinco cultivos seleccionados.

Se trazó la red de la tubería principal y secundaria, en los cinco tamaños de parcela, teniendo en cuenta la ubicación de la fuente de agua. Se estudió la operación del sistema y se diseñó la subunidad típica de riego por goteo en las condiciones más críticas de los cultivos, en las 25 combinaciones de parcelas. El caudal y volumen requeridos por metro de manguera y tiempo de riego se obtiene en función de la máxima evapotranspiración, espaciamiento entre laterales y goteros, número de 
goteros por planta y características del gotero. Luego, se determina el tamaño de la subunidad de riego y el caudal y se define el número de posiciones y rotaciones en cada turno de riego. Finalmente, se obtiene el caudal y horas de bombeo de todo el sistema (Bliesner y Keller, 2001). Por la presencia de sales superficiales y operación misma del riego por goteo, se hace necesario un lavado de sales (Bliesner y Keller, 2001).

Se prepararon datos de entrada para formular los 25 modelos de programación lineal: diámetros comerciales disponibles en el mercado, horas de bombeo anual, factor de recuperación del capital para un periodo de vida útil e interés bancario, costo anual de bombeo del sistema, caudales y presiones requeridas en cada nudo de la red, pérdidas de carga en los tramos de la tubería principal y secundaria. A partir de estos datos, se formularon los 25 modelos de programación lineal para la selección de los diámetros de tubería principal y secundaria que proporcionen los costos mínimos de implementos y operación del sistema.

Se diseñó el cabezal de riego considerando el caudal y pérdida de carga obtenida a la entrada de la red principal del sistema; así pudo definirse el tipo de bomba y motor, los filtros, las válvulas y los accesorios.
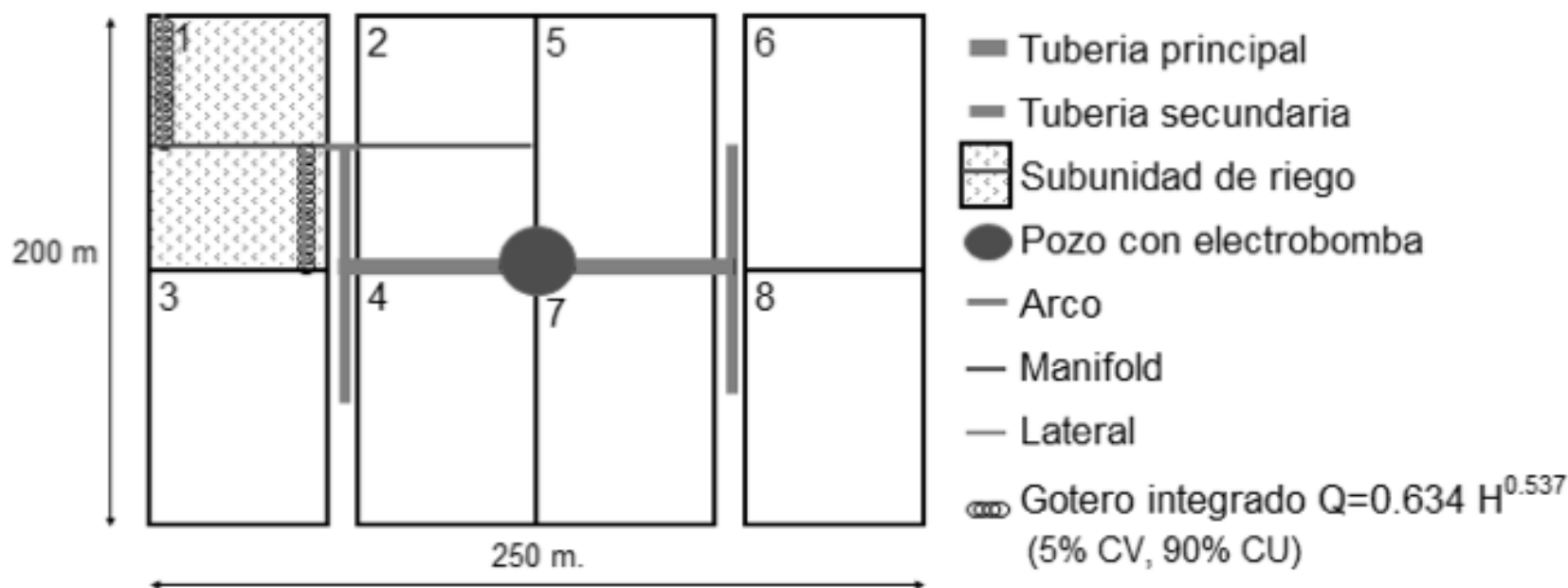

Figura 1. Trazo de la red de tubería principal y secundaria en parcela de 5 ha.

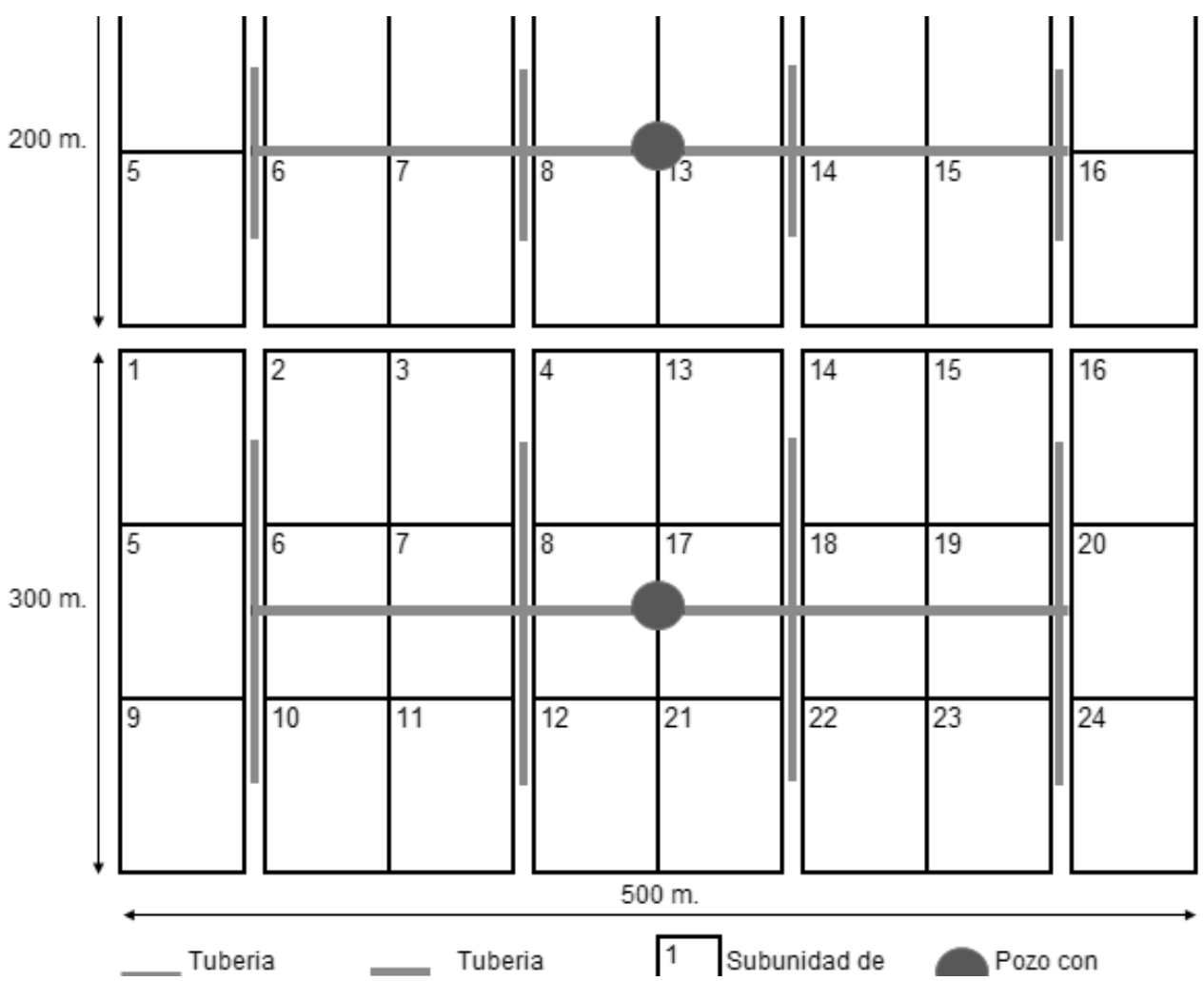

Figura 2. Trazo de la red de tubería principal y secundaria en parcela de 10 ha (panel superior) y 15 ha (panel inferior). 
Se realizó la evaluación económica con indicadores de rentabilidad VANE, TIRE y relación $\mathrm{B} / \mathrm{C}$ para un horizonte del proyecto de 10 años. Como parte de los costos del estudio, se consideraron los del equipo de riego (sistema de riego por goteo, aspersión, tubería de distribución, unidad de bombeo, cabezal de riego, accesorios); los de perforación y desarrollo del pozo; los de bombeo así como los de operación y mantenimiento del sistema. Para el procesamiento de la información se utilizaron los softwares CropWat para Windows, Excel con complemento "Solver", graficador Autocad y procesador de texto Word.

\section{Resultados y discusión}

\section{Trazo de la red de tubería principal y secundaria}

En las Figs. 1, 2 y 3 se aprecia el trazo de la red de tubería principal y secundaria para los tamaños de parcela seleccionadas $(5,10,15,20$ y 50 ha). Se consideró la perforación del pozo en el centro de la parcela para obtener una mejor distribución de presiones.

\section{Simulación de la operación del sistema}

Los cálculos realizados para definir la operación del sistema se presentan en las siguientes tablas: Evapotranspiración del cultivo, láminas de riego, tiempos de riego, horas de
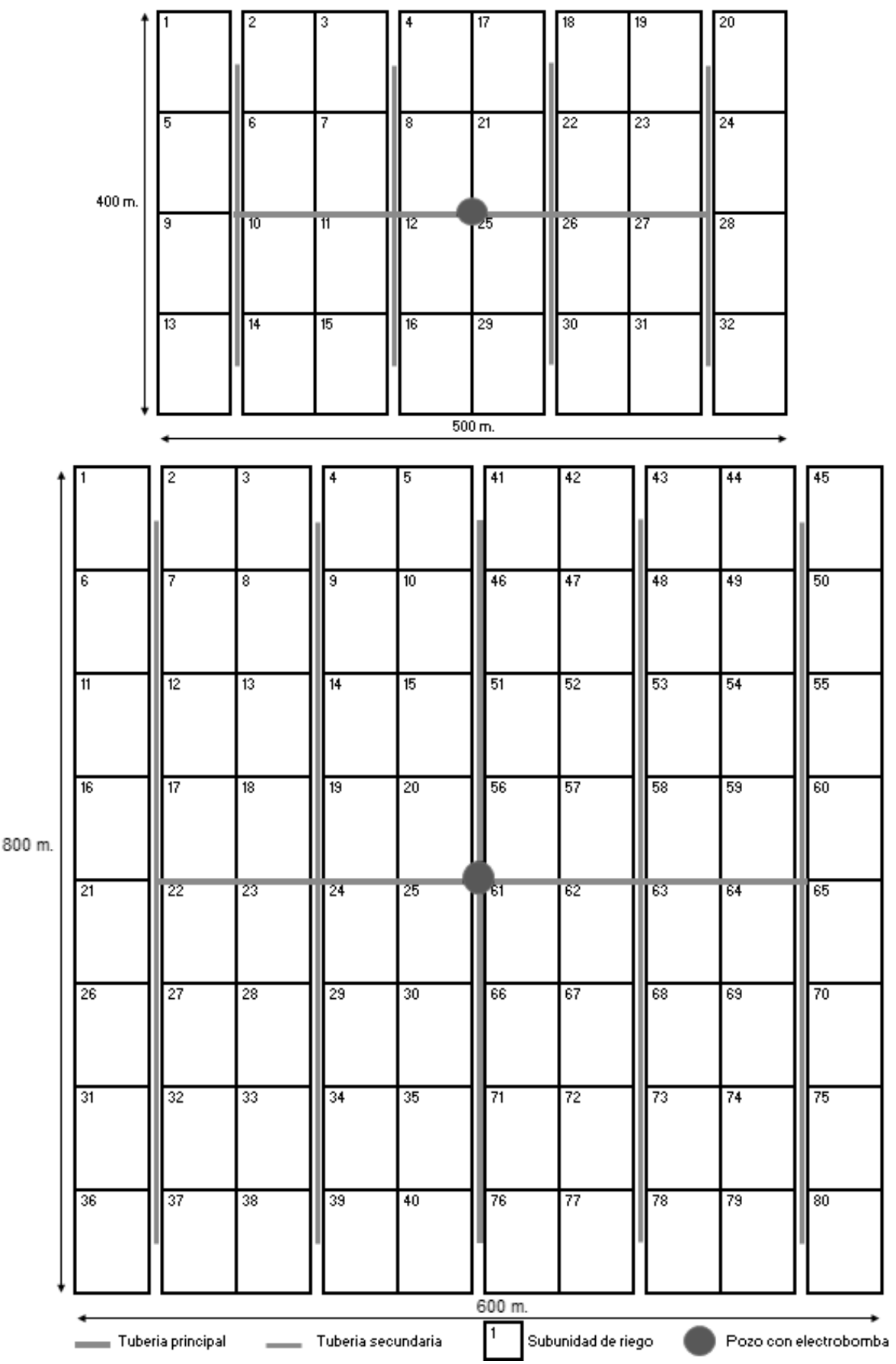

Figura 3. Trazo de la red de tubería principal y secundaria en parcela de 20 ha (panel superior) y 50 ha (panel inferior). 
Tabla 1. Evapotranspiración del cultivo, láminas de riego, tiempos de riego y horas de bombeo

\begin{tabular}{|c|c|c|c|c|c|c|c|c|c|c|c|c|c|}
\hline DETALLE & UNIDAD & ENE & FEB & MAR & ABR & MAY & JUN & JUL & $\mathrm{AGO}$ & SET & OCT & $\mathrm{NOV}$ & DIC \\
\hline Eto & $\mathrm{mm} / \mathrm{d}$ & 3,8 & 4,5 & 4,4 & 4,0 & 3,2 & 3,1 & 3,2 & 3,3 & 3,5 & 2,9 & 3,0 & 3,5 \\
\hline \multirow[t]{10}{*}{ Espárrago } & $\mathrm{Kc}$ & 0,8 & 0,8 & 08 & 0,8 & 0,8 & 0,8 & 0,8 & 0,8 & 0,8 & 0,8 & 0,8 & 0,8 \\
\hline & ETC mm/d & 2,9 & 34 & 3,3 & 30 & 2,4 & 2,3 & 2,4 & 2,5 & 2,6 & 2,2 & 2,3 & 2,6 \\
\hline & Lámina bruta mm/d & 3,2 & 3,8 &, 7 & 3,3 & 2,7 & 2,6 & 2,7 & 2,8 & 2,9 & 2,4 & 2,5 & 2,9 \\
\hline & Volumen Req $\mathrm{m}^{3} / \mathrm{ha}$ & 982 & 1050 & 1137 & 1000 & 827 & 775 & 827 & 853 & 875 & 749 & 750 & 904 \\
\hline & Volum req (1/d-m) & 6,3 & 7,5 & ,3 & 6,7 & 5,3 & 5,2 & 5,3 & 5,5 & 5,8 & 4,8 & 5,0 & 58 \\
\hline & $\mathrm{T}$ riego $(\mathrm{h} / \mathrm{d})$ & 1,1 & 1,3 & 1,2 & 1,1 & 09 & 0,9 & 0,9 & 0,9 & 1,0 & 0,8 & 0,8 & 1,0 \\
\hline & $\begin{array}{l}\text { T bombeo para } 50,20,10,5 \\
\text { ha }(\mathrm{h} / \mathrm{d})\end{array}$ & 8,4 & 10,0 & 98 & 89 & 7,1 & 6,9 & 7,1 & 7,3 & 7,8 & 6,4 & 6,7 & 7,8 \\
\hline & T bombeo para 15 ha $(\mathrm{h} / \mathrm{d})$ & 6,3 & 7,5 & 7,3 & 6,7 & 5,3 & 5,2 & 5,3 & 5,5 & 5,8 & 4,8 & 5,0 & 58 \\
\hline & $\begin{array}{l}\text { H de bombeo para 50,20,10, } \\
5 \text { ha }\end{array}$ & 262 & 280 & 303 & 267 & 220 & 207 & 220 & 227 & 233 & 200 & 200 & 241 \\
\hline & $\mathrm{H}$ de bombeo para 15 ha & 196 & 210 & 227 & 200 & 165 & 155 & 165 & 171 & 175 & 150 & 150 & 181 \\
\hline \multirow[t]{10}{*}{ Vid } & $\mathrm{Kc}$ & 0,6 & 0,6 & 0,8 & 0,6 & 0,4 & & & & 0,5 & 0,5 & 0,6 & 0,7 \\
\hline & $\mathrm{ETC} \mathrm{mm} / \mathrm{d}$ & 2,3 & 2,7 & 3,3 & 2,2 & 1,3 & & & & 1,6 & 1,5 & 1,8 & 2,3 \\
\hline & Lámina bruta mm/d & 2,5 & 30 & 3,7 & 1,7 & 10 & & & & 1,8 & 1,6 & 2,0 & 2,5 \\
\hline & Volumen Req m³/ha & 785 & 840 & 1137 & 5,0 & 2,3 & & & & 525 & 499 & 600 & 784 \\
\hline & Volum req (1/d-m) & 5,1 & 6,0 & 7,3 & 3,3 & 1,9 & & & & 3,5 & 3,2 & 4,0 & 5,1 \\
\hline & $\mathrm{T}$ riego $(\mathrm{h} / \mathrm{d})$ & 0,8 & 1,0 & 1,2 & 0,6 & 0,3 & & & & 0,6 & 0,5 & 0,7 & 0,8 \\
\hline & $\begin{array}{l}\text { T bombeo para } 50,20,10,5 \\
\text { ha }(h / d)\end{array}$ & 68 & 8,0 & 98 & 4,4 & 2,6 & & & & 4,7 & 4,3 & 5,3 & 6,7 \\
\hline & $\mathrm{T}$ bombeo para $15 \mathrm{ha}(\mathrm{h} / \mathrm{d})$ & 5,1 & 6,0 & 7,3 & 3,3 & 1,9 & & & &, 5 & 3,2 & 4,0 & 5,1 \\
\hline & $\begin{array}{l}\text { H de bombeo para } 50,20,10, \\
5 \text { ha }\end{array}$ & 209 & 224 & 303 & 132 & 79 & & & & 140 & 133 & 160 & 209 \\
\hline & $\mathrm{H}$ de bombeo para 15 ha & 157 & 168 & 227 & 99 & 60 & & & & 105 & 100 & 120 & 157 \\
\hline \multirow[t]{10}{*}{ Tomate } & $\mathrm{Kc}$ & 0,6 & & & 0,4 & 0,7 & 1,1 & 0,8 & 0,6 & 0,4 & 0,7 & 1,1 & 0,8 \\
\hline & $\mathrm{ETC} \mathrm{mm} / \mathrm{d}$ & 2,3 & & & 1,6 & 2,2 & 3,3 & 2,6 & 2,0 & 1,4 & 2,0 & 3,2 & 2,8 \\
\hline & Lámina bruta mm/d & 2,5 & & & 1,8 & 2,5 & 3,6 & 2,8 & 2,2 & 1,6 & 23 & 3,5 & 3,1 \\
\hline & Volumen Req m³/ha & 785 & & & 533 & 772 & 1085 & 882 & 682 & 467 & 699 & 1050 & 964 \\
\hline & Volum req $(1 / \mathrm{d}-\mathrm{m})$ & 5,1 & & & 3,6 & 5,0 & 7,2 & 5,7 & 4,4 & 3,1 & 4,5 & 7,0 & 6,2 \\
\hline & $\mathrm{T}$ riego $(\mathrm{h} / \mathrm{d})$ & 0,8 & & & 0,6 & 0,8 & 1,2 & 0,9 & 0,7 & 0,5 & 0,8 & 1,2 & 1,0 \\
\hline & $\begin{array}{l}\text { T bombeo para } 50,20,10,5 \\
\text { ha }(\mathrm{h} / \mathrm{d})\end{array}$ & 6,8 & & & 4,7 & 66 & 9,6 & 7,6 & 5,9 & 4,1 & 6,0 & 9,3 & 8,3 \\
\hline & $\mathrm{T}$ bombeo para $15 \mathrm{ha}(\mathrm{h} / \mathrm{d})$ & 5,1 & & & 3,6 & 50 & 7,2 & 7 & 4,4 & 3,1 & 4,5 & 7,0 & 6,2 \\
\hline & $\begin{array}{l}\text { H de bombeo para } 50,20,10 \text {, } \\
5 \text { ha }\end{array}$ & 209 & & & 142 & 206 & 289 & 235 & 182 & 124 & 186 & 280 & 257 \\
\hline & $\mathrm{H}$ de bombeo para 15 ha & 157 & & & 107 & 154 & 217 & 176 & 136 & 93 & 140 & 210 & 193 \\
\hline \multirow[t]{14}{*}{ Ají Páprika } & $\mathrm{Kc}$ & & & & 0,4 & 0,7 & 0,9 & 1,0 & 0,9 & 0,8 & 0,6 & 0,5 & \\
\hline & $\mathrm{ETC} \mathrm{mm} / \mathrm{d}$ & & & & 16 & 2,2 & 2,8 & 3,2 & 3,0 & 2,8 & 1,7 & 1,4 & \\
\hline & Lámina bruta mm/d & & & & 18 & 2,5 & 3,1 & 3,6 & 3,3 & 3,1 & 1,9 & 1,6 & \\
\hline & Volumen Req m³/ha & & & & 533 & 772 & 930 & 1102 & 1023 & 933 & 599 & 470 & \\
\hline & Volum req (1/d-m) & & & & 1,8 & 2,5 & 3,1 & 3,6 & 3,3 & 3,1 & 1,9 & 1,6 & \\
\hline & T riego $(\mathrm{h} / \mathrm{d})$ & & & & 0,3 & 0,4 & 0,5 & 0,6 & 0,6 & 0,5 & 0,3 & 0,3 & \\
\hline & $\mathrm{T}$ bombeo para $50 \mathrm{ha}(\mathrm{h} / \mathrm{d})$ & & & & 5,9 & 8,3 & 10,3 & 11,9 & 11,0 & 10,44 & 6,4 & 5,2 & \\
\hline & $\mathrm{T}$ bombeo para $20,10 \mathrm{ha}(\mathrm{h} / \mathrm{d})$ & & & & 4,7 & 6,6 & 8,3 & 9,5 & 8,8 & 8,3 & 5,2 & 4,2 & \\
\hline & $\mathrm{T}$ bombeo para $15 \mathrm{ha}(\mathrm{h} / \mathrm{d})$ & & & & 3,6 & 5,0 & 6,2 & 7,1 & 6,6 & 6,2 & 3,9 & 3,1 & \\
\hline & $\mathrm{T}$ bombeo para 5 ha $(\mathrm{h} / \mathrm{d})$ & & & & 2,4 & 3,3 & 4,1 & 47 & 4,4 & 4,1 & 2,6 & 21 & \\
\hline & $\mathrm{H}$ de bombeo para 50 ha & & & & 178 & 257 & 310 & 367 & 341 & 311 & 200 & 157 & \\
\hline & H de bombeo para 20,10 ha & & & & 142 & 206 & 248 & 294 & 273 & 249 & 160 & 125 & \\
\hline & $\mathrm{H}$ de bombeo para 15 ha & & & & 107 & 154 & 186 & 220 & 205 & 187 & 120 & 94 & \\
\hline & $\mathrm{H}$ de bombeo para 5 ha & & & & 71 & 103 & 124 & 147 & 136 & 124 & 80 & 63 & \\
\hline
\end{tabular}




\begin{tabular}{|c|c|c|c|c|c|c|c|c|c|c|c|c|c|}
\hline \multirow[t]{14}{*}{ Cebolla } & $\mathrm{Kc}$ & 0,5 & 0,4 & 0,7 & 0,9 & 1,0 & 0,6 & 0,5 & 0,4 & 0,7 & 0,9 & 1,0 & 0,6 \\
\hline & ETC $\mathrm{mm} / \mathrm{d}$ & 1,8 & 1,7 & 3,0 & 3,6 & 32 & 1,9 & 1,5 & 1,2 & 2,4 & 2,6 & 3,0 & 2,1 \\
\hline & Lámina bruta mm/d & 2,0 & 1,9 & 3,3 & 4,0 & 3,6 & 2,1 & 1,7 & 1,4 & 2,6 & 2,9 & 3,3 & 2,3 \\
\hline & Volumen Req $\mathrm{m}^{3} / \mathrm{ha}$ & 615 & 518 & 1031 & 1200 & 1102 & 620 & 518 & 421 & 793 & 899 & 1000 & 723 \\
\hline & Volum req (1/d-m) & 2,0 & 1,9 & 3,3 & 40 & 3,6 & 21 & 1,7 & 1,4 & 2,6 & 2,9 & 3,3 & 2,3 \\
\hline & T riego $(\mathrm{h} / \mathrm{d})$ & 0,3 & 0,3 & 0,6 & 0,7 & 0,6 & 03 & 0,3 & 0,2 & 0,4 & 0,5 & 06 & 0,4 \\
\hline & $\mathrm{T}$ bombeo para 50 ha $(\mathrm{h} / \mathrm{d})$ & 6,6 & 6,2 & 11.1 & 13,3 & 11,9 & 6,9 & 56 & 4,5 & 8,8 & 9,7 & 11,1 & 7,8 \\
\hline & $\mathrm{T}$ bombeo para 20,10 ha $(\mathrm{h} / \mathrm{d})$ & 5,3 & 4,9 & 8,9 & 10,7 & 9,5 & 5,5 & 4,5 & 3,6 & 7,1 & 7,7 & 8,9 & 6,2 \\
\hline & $\mathrm{T}$ bombeo para 15 ha $(\mathrm{h} / \mathrm{d})$ & 4,0 & 3,7 & 66 & 8,0 & 7,1 & 4,1 & 3,3 & 2,7 & 5,3 & 5,8 & 6,7 & 4,7 \\
\hline & $\mathrm{T}$ bombeo para $5 \mathrm{ha}(\mathrm{h} / \mathrm{d})$ & 2,6 & 2,5 & 4,4 & 5,3 & 4,7 & 2,8 & 2,2 & 1,8 & 3,5 & 3,9 & 4,4 & 3,1 \\
\hline & $\mathrm{H}$ de bombeo para 50 ha & 205 & 173 & 344 & 400 & 367 & 207 & 173 & 140 & 264 & 300 & 333 & 241 \\
\hline & H de bombeo para 20,10 ha & 164 & 138 & 275 & 320 & 294 & 165 & 138 & 112 & 212 & 240 & 267 & 193 \\
\hline & H de bombeo para 15 ha & 123 & 104 & 206 & 240 & 220 & 124 & 104 & 84 & 159 & 180 & 200 & 145 \\
\hline & $\mathrm{H}$ de bombeo para 5 ha & 82 & 69 & 137 & 160 & 147 & 83 & 69 & 56 & 106 & 120 & 133 & 96 \\
\hline
\end{tabular}

* Estación meteorológica PE Hacienda del Sur.

bombeo mensual y anual según tipo de cultivo y tamaño de parcela, para una eficiencia de riego del $90 \%$ (Tabla 1); Caudal del sistema y tiempo de bombeo según cultivo y tamaño de parcela (Tabla 2); Tamaño y caudal de subunidad de riego: lateral, manifold y arco (Tabla 3 ).

Finalmente, en la Tabla 4 y en las Figuras de la 4 a la 8 , se presenta el número de subunidades que se riegan en forma simultánea y el tiempo de riego diario en las condiciones más críticas.

Tabla 2. Caudal del sistema y tiempo de bombeo

\begin{tabular}{cccccccccccc}
\hline $\begin{array}{c}\text { Área } \\
\text { (ha) }\end{array}$ & $\begin{array}{c}\mathrm{N}^{\circ} \\
\text { subun }\end{array}$ & \multicolumn{2}{c}{ Espárrago } & \multicolumn{2}{c}{ Vid } & \multicolumn{2}{c}{ Tomate } & \multicolumn{2}{c}{ Ají Páprika } & \multicolumn{2}{c}{ Cebolla } \\
\hline 50 & 80 & 50 & 10 & 50 & 10 & 50 & 10 & 40 & 12 & 40 & 13 \\
20 & 32 & 20 & 10 & 20 & 10 & 20 & 10 & 20 & 9 & 20 & 11 \\
15 & 24 & 20 & 8 & 20 & 7 & 20 & 7 & 20 & 7 & 20 & 8 \\
10 & 16 & 10 & 10 & 10 & 10 & 10 & 10 & 10 & 9 & 10 & 11 \\
5 & 8 & 5 & 10 & 5 & 10 & 5 & 10 & 10 & 5 & 10 & 5 \\
\hline
\end{tabular}

Tabla 3. Tamaño y caudal de subunidad de riego en parcelas de 5, 10, 15, 20 y 50 ha: lateral, manifold y arco

\begin{tabular}{|c|c|c|c|c|c|c|c|c|c|c|}
\hline \multicolumn{2}{|l|}{ Detalle } & \multirow{2}{*}{$\begin{array}{c}\text { Espárrago } \\
\text { Vid }\end{array}$} & \multirow{2}{*}{$\begin{array}{c}\text { Tomate } \\
2\end{array}$} & \multirow{2}{*}{$\begin{array}{l}\text { Páprika } \\
\text { Cebolla } \\
2\end{array}$} & \multirow{2}{*}{\multicolumn{2}{|c|}{$\begin{array}{c}\text { Detalle } \\
=\text { longitud }\end{array}$}} & \multicolumn{2}{|c|}{ EspárragoVid } & \multirow{2}{*}{$\begin{array}{c}\text { Tomate } \\
50\end{array}$} & \multirow{2}{*}{$\begin{array}{c}\begin{array}{c}\text { Páprika } \\
\text { Cebolla }\end{array} \\
50\end{array}$} \\
\hline Gotero & $1 / \mathrm{h}$ & & & & & & $\mathrm{m}$ & 50 & & \\
\hline Esp. Laterales & $\mathrm{m}$ & 2 & 2 & 1 & एँ & diámet & $\mathrm{mm}$ & 16 & 16 & 16 \\
\hline Esp. Goteros & got $/ \mathrm{m}$ & 3 & 3 & 3 & 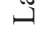 & caudal & $1 / \mathrm{s}$ & 0,08 & 0,08 & 0,08 \\
\hline Esp. Plantas & got/plant & 2 & 1 & 2 & 흠 & longitud & $\mathrm{m}$ & 60 & 60 & 60 \\
\hline Área subunida & ha & 0,6 & 0,6 & 0,6 & $\stackrel{0}{\Xi}$ & diámet & pulg & 2 & 2 & 3 \\
\hline largo & $\mathrm{m}$ & 100 & 100 & 100 & $\sum^{\pi}$ & caudal & $1 / \mathrm{s}$ & 5 & 5 & 10 \\
\hline ancho & $\mathrm{m}$ & 60 & 60 & 60 & & longitud & $\mathrm{m}$ & 3 & 3 & 3 \\
\hline \multirow[t]{3}{*}{ caudal } & $1 / \mathrm{s}$ & 5 & 5 & 10 & 8 & diámet & pulg & 2 & 2 & 3 \\
\hline & & & & & 光 & caudal & $1 / \mathrm{s}$ & 5 & 5 & 10 \\
\hline & & & & & & presión* & $\mathrm{m}$ & 13,69 & 13,69 & 12,63 \\
\hline
\end{tabular}

Tabla 4. Operación del sistema

\begin{tabular}{|c|c|c|c|c|c|c|c|c|c|c|}
\hline \multirow{2}{*}{$\frac{\text { Cultivo }}{\text { Tamaño de parcela (ha) }}$} & \multicolumn{5}{|c|}{ Espárrago, Vid, Tomate } & \multicolumn{5}{|c|}{ Páprika, Cebolla } \\
\hline & 50 & 20 & 15 & 10 & 5 & 50 & 20 & 15 & 10 & 5 \\
\hline Posiciones de riego/día & 10 & 4 & 4 & 2 & 1 & 4 & 2 & 2 & 1 & 1 \\
\hline $\mathrm{N}^{\circ}$ de rotaciones & 8 & 8 & 6 & 8 & 8 & 20 & 16 & 12 & 16 & 8 \\
\hline $\mathrm{N}^{\circ}$ de subunidades/riego simultáneo & 10 & 4 & 4 & 2 & 1 & 4 & 2 & 2 & 1 & 1 \\
\hline
\end{tabular}




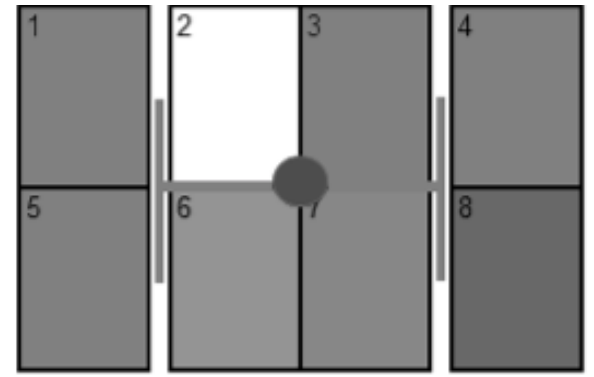

Figura 4. Turnos de riego en parcela de 5 ha: 1 subunidad por riego simultáneo.

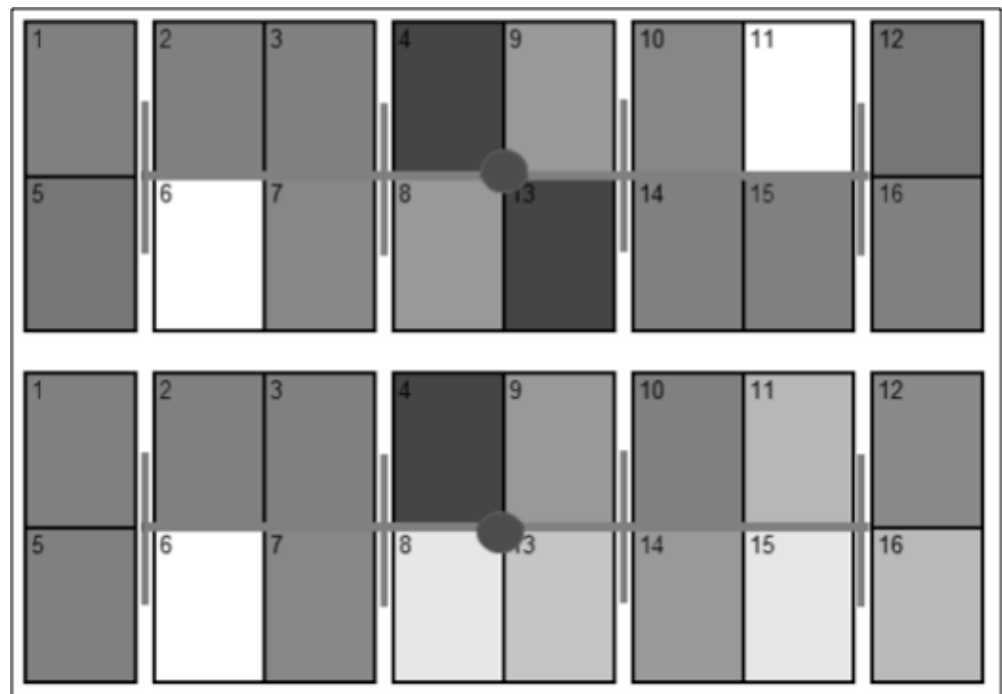

Figura 5. Turnos de riego en parcela de 10 ha. Panel superior: 2 subunidades por riego simultáneo en espárrago, vid y tomate. Panel inferior: 1 subunidad por riego simultáneo en páprika y cebolla.
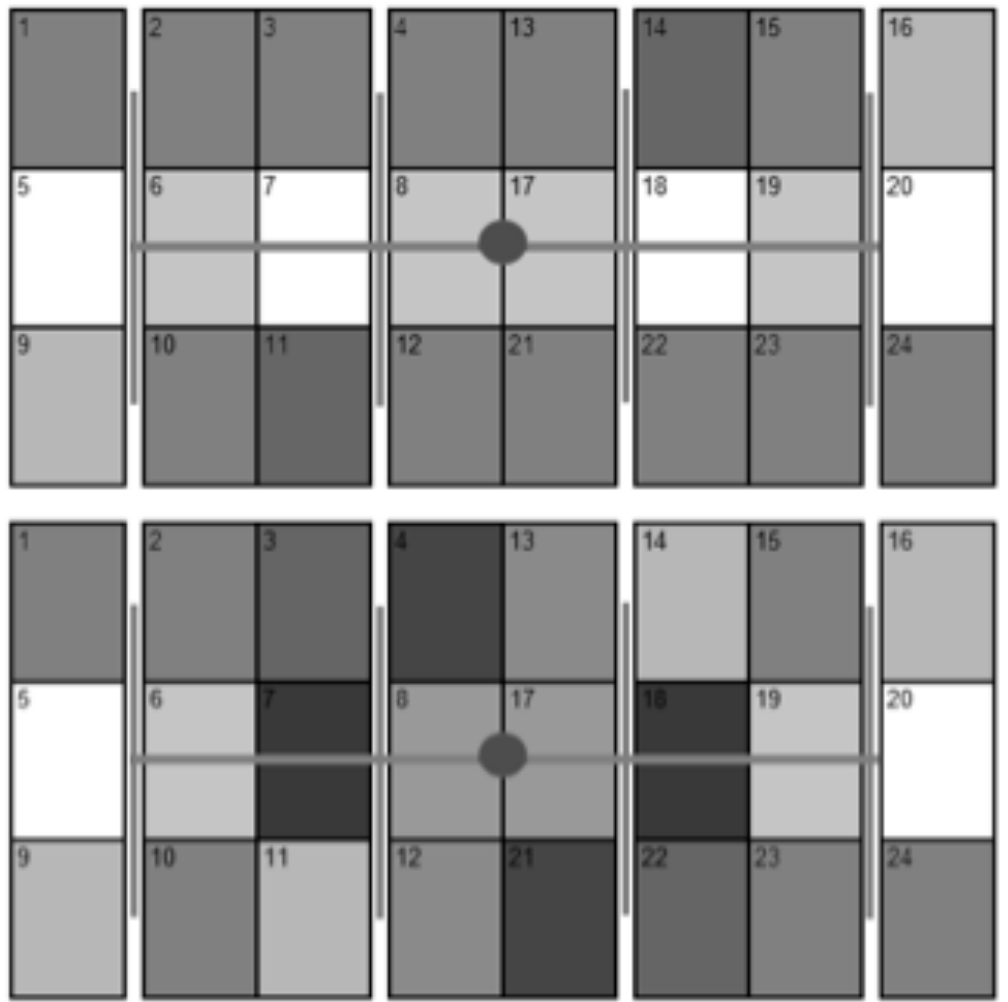

Figura 6. Turnos de riego en parcela de 15 ha. Panel superior: 4 subunidades con espárrago, vid y tomate. Panel inferior: 2 subunidades con páprika y cebolla. 


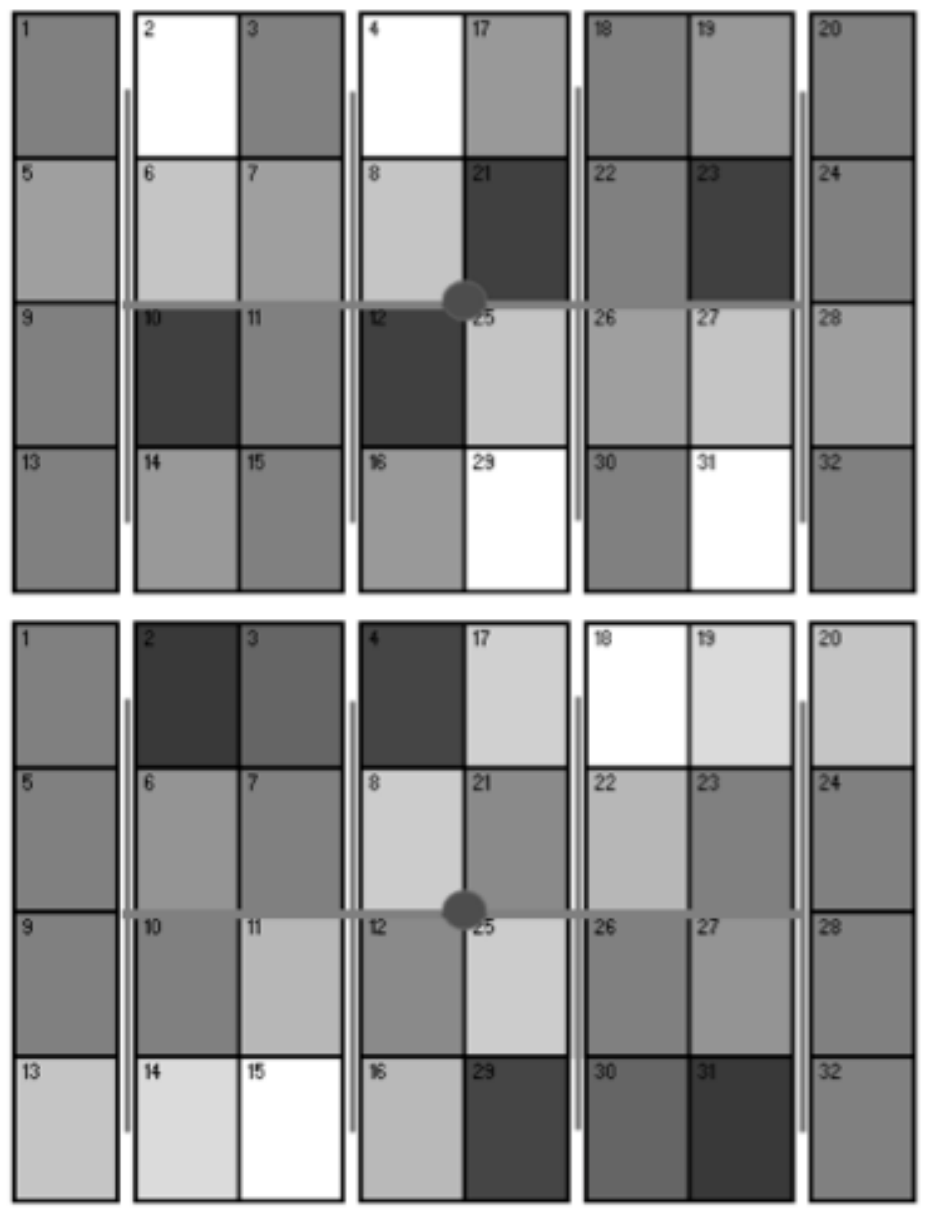

Figura 7. Turnos de riego en parcela de 20 ha. Panel superior: 4 subunidades con espárrago, vid y tomate. Panel inferior: 2 subunidades con páprika y cebolla.
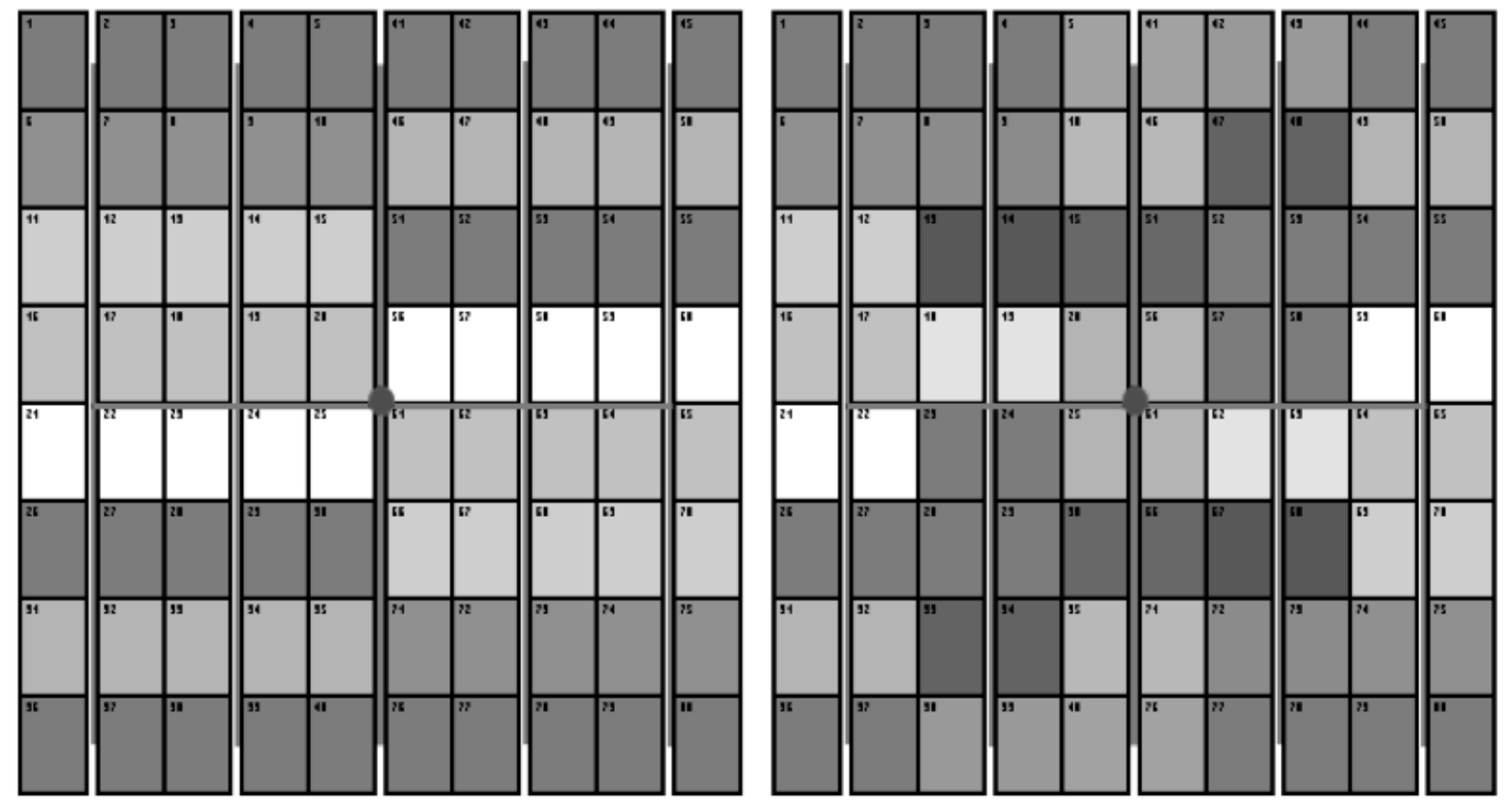

Figura 8. Turnos de riego en parcela de 50 ha. Panel superior: 10 subunidades con espárrago, vid y tomate. Panel inferior: 4 subunidades con páprika y cebolla. 
Se diseñó el sistema de riego por aspersión para aplicar la lámina de lavado de sales; por lo que se compatibilizó el diseño del sistema de aspersión con el diseño del sistema de riego por goteo en las 25 combinaciones de parcelas del estudio. El aspersor elegido fue el Naam de presión 15 $\mathrm{m}$ y caudal $1,44 \mathrm{~m}^{3} / \mathrm{h}$ en líneas de mangueras de PEMD de $25 \mathrm{~mm}$ de diámetro con $50 \mathrm{~m}$ de longitud, conectados al manifold a nivel de subunidad de riego, a través de hidrantes de acople rápido con salidas de 3/4“. La lámina de lavado de sales se obtuvo con la conductividad eléctrica al $75 \%$ de producción de los cultivos, aplicando la ecuación propuesta por Coachelli (Bliesner y Keller, 2001). Con ayuda del regulador de presión Plastro GVAT en el arco de riego, el sistema está preparado para accionar aspersores de $15 \mathrm{~m}$ de presión nominal.

Datos de entrada para formular el modelo de Programación Lineal.

Los diámetros comerciales disponibles en el mercado son de 2", 3", 4", 6" y 8" con costos que se detallan en la Tabla 5 .
El factor de recuperación del capital es igual a $F R C=\frac{i(1+i)^{n}}{(1+i)^{n}-1}=0.1468$ paraunperiododevidaútilde 10 años (n) e interés bancario del $10 \%$ (r). El costo anual de bombeo del sistema es igual a:

$$
C=C_{\text {energia }}+C_{\text {bomba }}=\frac{C_{\text {Villouri }}}{0.93 h p-h r / k w-h r} \frac{Q^{*} t}{76^{*} E}+C_{\text {bomba }}
$$

Donde: $\mathrm{C}_{\text {Villacuri }}$ es el costo de energía en la zona de estudio, $0.0769 \$ / \mathrm{kw}-\mathrm{h}$ (agosto 2004); $\mathrm{C}_{\text {bomba }}$ es el costo de la bomba $\mathrm{Cb}=12233+95 \mathrm{Ht}$ (Hidrostal); Q es el caudal total del sistema (1/s) de la Tabla 2; t son las horas de bombeo anual; E es la eficiencia de la bomba*eficiencia del motor, $70 \%$ y $90 \%$ respectivamente (Hidrostal).

Se tuvo en cuenta la simetría de la red y turnos de riego en la selección de los tramos de tubería de la red de distribución. Así, se tuvo 4, 3, 3, 3 y 1 tramos en las parcelas de $50,20,15,10$ y 5 ha respectivamente, que se aprecia en la Fig. 9. Las pérdidas de carga en la red de distribución se detallan en la Tabla 6 , por tramos de la red, según la simetría del sistema.

Tabla 5. Costo de tuberías anualizadas

\begin{tabular}{rrrrrrr}
\hline $\mathrm{N}^{\circ}$ & \multicolumn{2}{c}{$\begin{array}{c}\text { Diámetro } \\
\text { exterior }\end{array}$} & $\begin{array}{c}\text { Espesor } \\
\text { Pulg. }\end{array}$ & $\begin{array}{c}\text { Diámetro } \\
\text { interior mm }\end{array}$ & \multicolumn{1}{c}{ C. Unitario } & C. Anualiz \\
\hline 1 & 2 & 60 & 1,8 & 58,2 & 1,38 & 0,18 \\
2 & 3 & 89 & 2,2 & 84,1 & 2,08 & 0,27 \\
3 & 4 & 114 & 2,8 & 106,4 & 3,41 & 0,45 \\
4 & 6 & 168 & 4,1 & 159,8 & 7,35 & 0,97 \\
5 & 8 & 219 & 5,3 & 208,4 & 12,38 & 1,63 \\
\hline
\end{tabular}

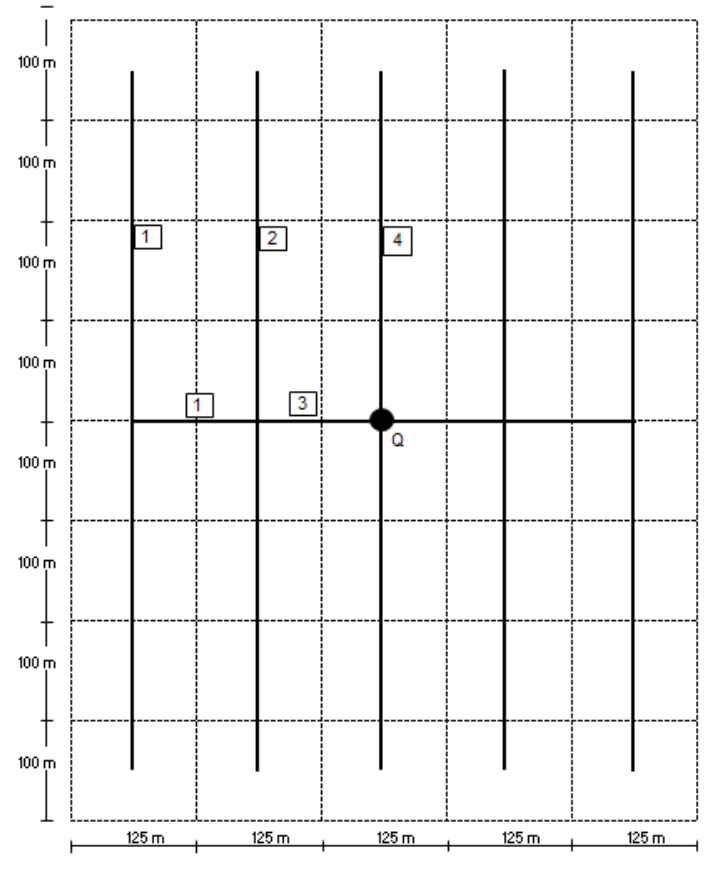

$50 \mathrm{Ha}$

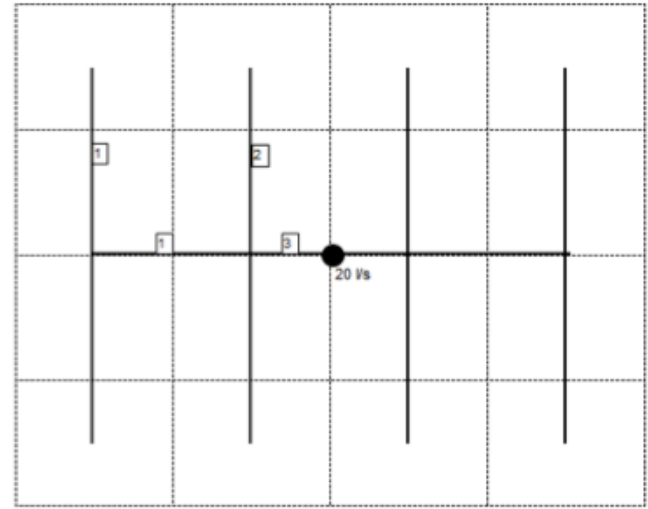

$20 \mathrm{Ha}$

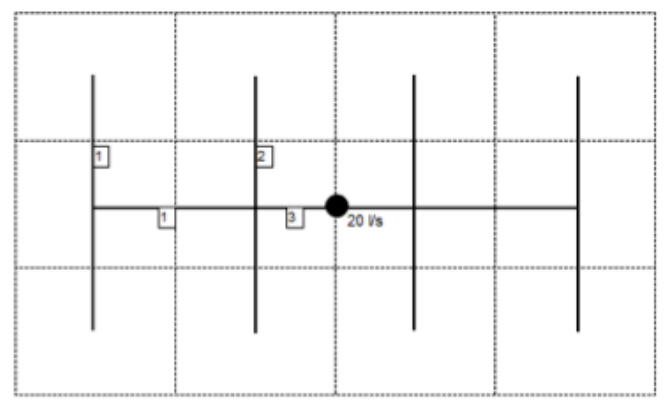

$15 \mathrm{Ha}$ 


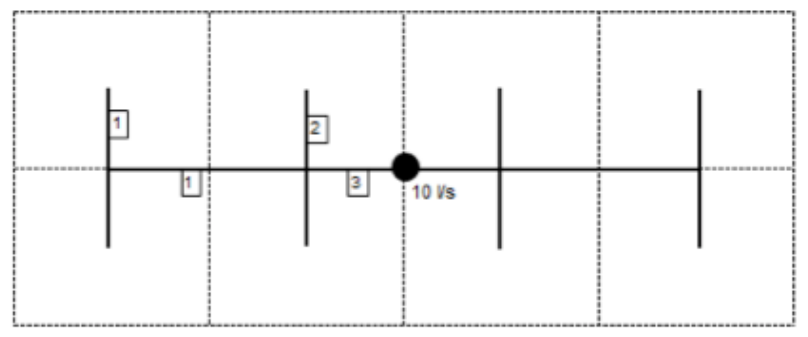

$10 \mathrm{Ha}$

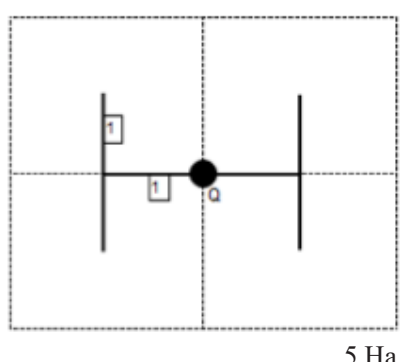

$5 \mathrm{Ha}$

Figura 9. Tramos de tubería según simetría y turnos de riego en la red de distribución.

\section{Modelos de programación lineal}

Se formularon 25 modelos de programación lineal según tamaño de parcela y cultivos seleccionados; con variables de decisión en la presión de operación de la bomba $(\mathrm{Ht})$ y longitudes de los tramos de tubería (Lij), donde i toma valores de 1, 2, 3 y 4 y j toma valores de 2", 3", 4", 6" y 8" pulgadas, respectivamente.

Tabla 6. Pérdidas por fricción (m/m) en 50 ha(a), 20 y 15 ha(b), 10 ha(c) y 5 ha(d)

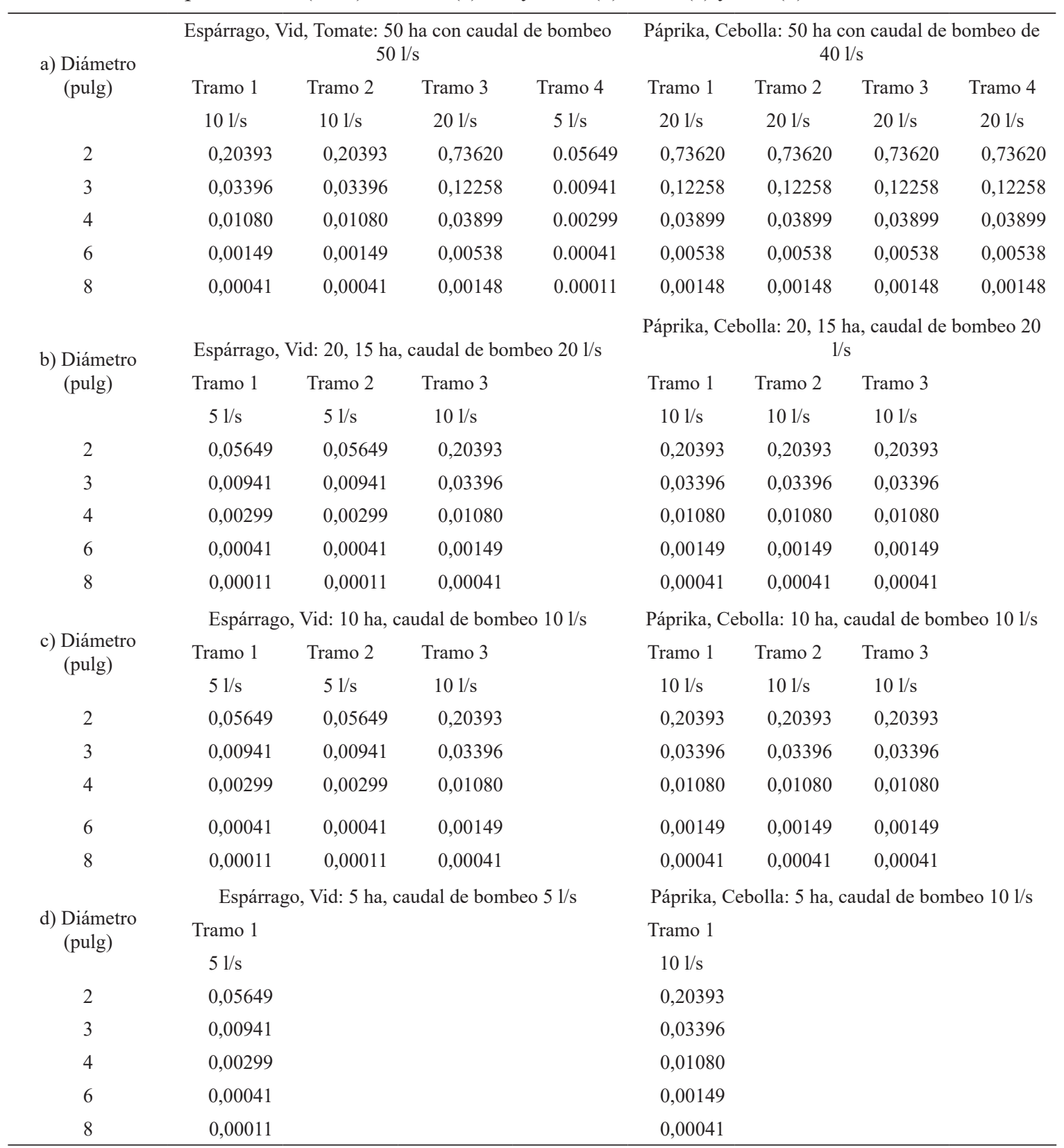


El modelo de programación lineal, en cebolla, con 50 ha es: $\mathrm{Z}=258,4 \mathrm{Ht}+0,182 \mathrm{~L} 11+0,273 \mathrm{~L} 12+0,448 \mathrm{~L} 13+$ $0,966 \mathrm{~L} 14+1,628 \mathrm{~L} 15+0,182 \mathrm{~L} 21+0,273 \mathrm{~L} 22+0,448 \mathrm{~L} 23+$ $0,966 \mathrm{~L} 24+1,628 \mathrm{~L} 25+0,182 \mathrm{~L} 31+0,273 \mathrm{~L} 32+0,448 \mathrm{~L} 33+$ $0,966 \mathrm{~L} 34+1,628 \mathrm{~L} 35+0,182 \mathrm{~L} 41+0,273 \mathrm{~L} 42+0,448 \mathrm{~L} 43+$ $0,966 \mathrm{~L} 44+1,628 \mathrm{~L} 45 ;$ Ht- 0,7362L11- 0,1226L120,0390L13- 0,0054 L14- 0,0015 L15- 0,7362 L31$0,1226 \mathrm{~L} 32-\quad 0,0390 \mathrm{~L} 33-\quad 0,0054 \mathrm{~L} 34-\quad 0,0015 \mathrm{~L} 35 \geq$ 1,63 ; Ht- 0,7362L21- 0,1226L22- 0,0390L23$0,0054 \mathrm{~L} 24-\quad 0,0015 \mathrm{~L} 25-\quad 0,7362 \mathrm{~L} 31-\quad 0,1226 \mathrm{~L} 32-$ $0,0390 \mathrm{~L} 33-0,0054 \mathrm{~L} 34-0,0015 \mathrm{~L} 35 \geq 12,63$; Ht- 0,7362L41- 0,1226L42- 0,0390L43- 0,0054L44$0,0015 \mathrm{~L} 45 \geq 12,63 ; \mathrm{L} 11+\mathrm{L} 12+\mathrm{L} 13+\mathrm{L} 14+\mathrm{L} 15=475$; $\mathrm{L} 21+\mathrm{L} 22+\mathrm{L} 23+\mathrm{L} 24+\mathrm{L} 25=350$; L31+L32+L33+L34+L35=125; L41+L42+L43+L44+L45 $=350$.

En el caso de cebolla con 20 ha es: $Z=111,7 \mathrm{Ht}+0,182 \mathrm{~L} 11+$ $0,273 \mathrm{~L} 12+0,448 \mathrm{~L} 13+0,966 \mathrm{~L} 14+1,628 \mathrm{~L} 15+0,182 \mathrm{~L} 21$ $+0,273 \mathrm{~L} 22+0,448 \mathrm{~L} 23+0,966 \mathrm{~L} 24+1,628 \mathrm{~L} 25+$ $0,182 \mathrm{~L} 31+0,273 \mathrm{~L} 32+0,448 \mathrm{~L} 33+0,966 \mathrm{~L} 34+1,628 \mathrm{~L} 35$; Ht - 0,2039L11 - 0,0340L12 - 0,0108L13 - 0,0015L14 $-0,0004 \mathrm{~L} 15-0,2039 \mathrm{~L} 31-0,0340 \mathrm{~L} 32-0,0108 \mathrm{~L} 33$ $-0,0015 \mathrm{~L} 34-0,0004 \mathrm{~L} 35 \geq 12,63 ; \mathrm{Ht}-0,2039 \mathrm{~L} 21$ $-0,0340 \mathrm{~L} 22-0,0108 \mathrm{~L} 23-0,0015 \mathrm{~L} 24-0,0004 \mathrm{~L} 25$ $-0,2039$ L31 - 0,0340L32 - 0,0108L33 - 0,0015L34 $0,0004 \mathrm{~L} 35 \geq 12,63 ; \mathrm{L} 11+\mathrm{L} 12+\mathrm{L} 13+\mathrm{L} 14+\mathrm{L} 15=275$; $\mathrm{L} 21+\mathrm{L} 22+\mathrm{L} 23+\mathrm{L} 24+\mathrm{L} 25=150$;

$\mathrm{L} 31+\mathrm{L} 32+\mathrm{L} 33+\mathrm{L} 34+\mathrm{L} 35=62.5$.
Para la cebolla con 15 ha es: $\mathrm{Z}=72,8 \mathrm{Ht}+0,182 \mathrm{~L} 11$ $+0,273 \mathrm{~L} 12+0,448 \mathrm{~L} 13+0,966 \mathrm{~L} 14+1,628 \mathrm{~L} 15+$ $0,182 \mathrm{~L} 21+0,273 \mathrm{~L} 22+0,448 \mathrm{~L} 23+0,966 \mathrm{~L} 24+1,628 \mathrm{~L} 25+$ $0,182 \mathrm{~L} 31+0,273 \mathrm{~L} 32+0,448 \mathrm{~L} 33+0,966 \mathrm{~L} 34+1,628 \mathrm{~L} 35$; Ht- 0,2039L11- 0,0340L12- 0,0108L13-0,0015L140,0004L15- 0,2039L31- 0,0340L32- 0,0108L330,0015L34-0,0004L35 $\geq 12,63$; Ht-,2039L21-0,0340L220,0108L23-0,0015L24-0,0004L25- 0,2039L31 - 0,0340L32- 0,0108L33- 0,0015L34- 0,0004L35 $\geq \quad 12,63 ; \quad \mathrm{L} 11+\mathrm{L} 12+\mathrm{L} 13+\mathrm{L} 14+\mathrm{L} 15=225 ;$ $\mathrm{L} 21+\mathrm{L} 22+\mathrm{L} 23+\mathrm{L} 24+\mathrm{L} 25=100$; $\mathrm{L} 31+\mathrm{L} 32+\mathrm{L} 33+\mathrm{L} 34+\mathrm{L} 35=62,5$.

El modelo para cebolla con 10 ha es : $\mathrm{Z}=92,2 \mathrm{Ht}+$ $0,182 \mathrm{~L} 11+0,273 \mathrm{~L} 12+0,448 \mathrm{~L} 13+0,966 \mathrm{~L} 14+1,628 \mathrm{~L} 15$ $+0,182 \mathrm{~L} 21+0,273 \mathrm{~L} 22+0,448 \mathrm{~L} 23+0,966 \mathrm{~L} 24+$ $1,628 \mathrm{~L} 25+0,182 \mathrm{~L} 31+0,273 \mathrm{~L} 32+0,448 \mathrm{~L} 33+0,966 \mathrm{~L} 34$ $+1,628 \mathrm{~L} 35 ; \mathrm{Ht}-0,2039 \mathrm{~L} 11-0,0340 \mathrm{~L} 12-0,0108 \mathrm{~L} 13$ $-0,0015 \mathrm{~L} 14-0,0004 \mathrm{~L} 15-0,2039 \mathrm{~L} 31-0,0340 \mathrm{~L} 32$ $-0,0108 \mathrm{~L} 33-0,0015 \mathrm{~L} 34-0,0004 \mathrm{~L} 35 \geq 12,63$; Ht 0,2039L21 - 0,0340L22 - 0,0108L23 - 0,0015L24 $0,0004 \mathrm{~L} 25$

- 0,2039L31 - 0,0340L32 - 0,0108L33 $0,0015 \mathrm{~L} 34-0,0004 \mathrm{~L} 35 \geq 12,63 ; \quad \mathrm{L} 11+\mathrm{L} 12+\mathrm{L} 13+$ $\mathrm{L} 14+\mathrm{L} 15=175 ; \quad \mathrm{L} 21+\mathrm{L} 22+\mathrm{L} 23+\mathrm{L} 24+\mathrm{L} 25=50$; $\mathrm{L} 31+\mathrm{L} 32+\mathrm{L} 33+\mathrm{L} 34+\mathrm{L} 35=62,5$.

En las Figuras 10, 11, 12 y $\mathbf{1 3}$ se detalla para el resto de cultivos en 50, 20, 15 y 10 ha, respectivamente.

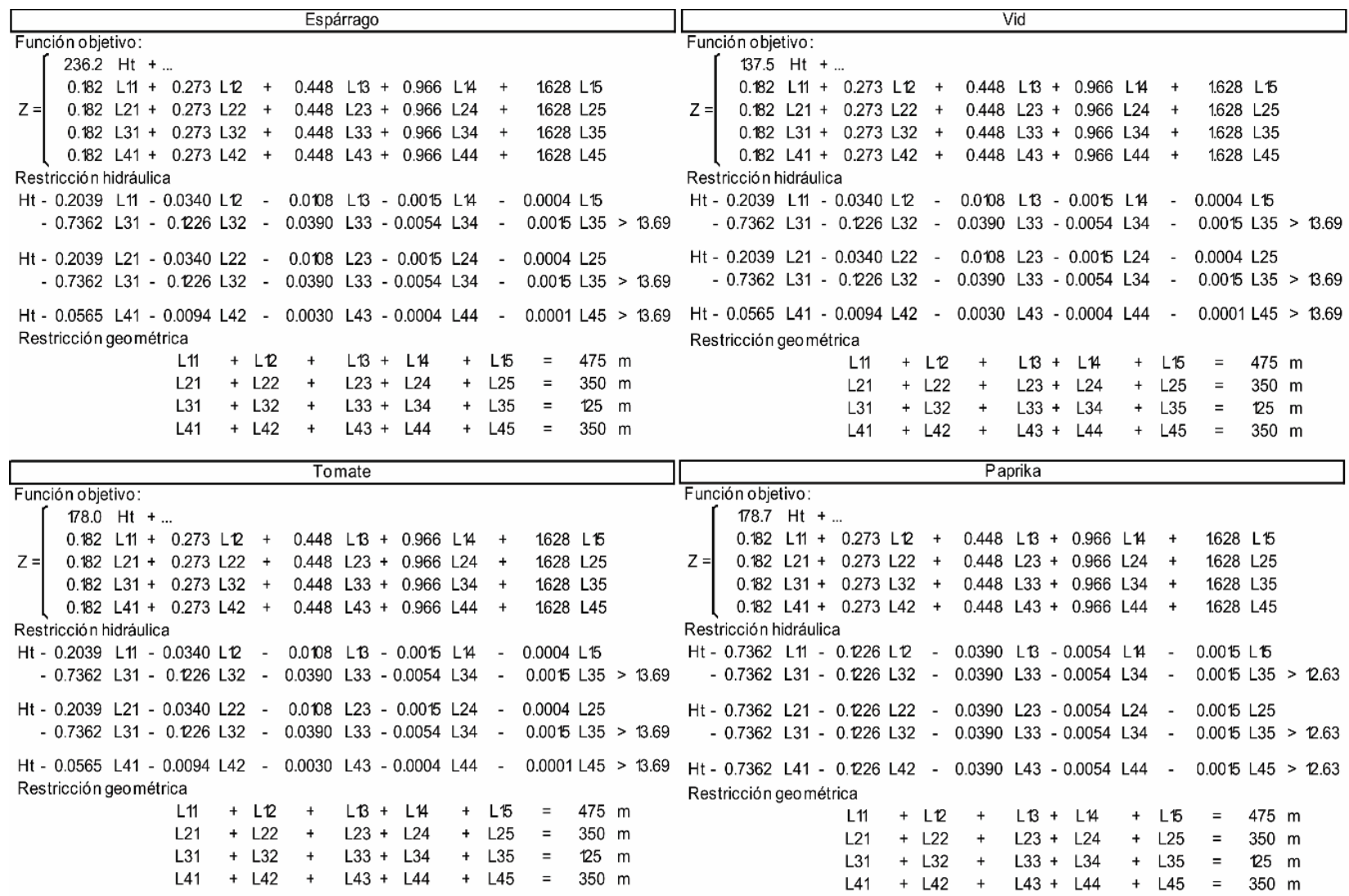

Figura 10. Modelos de programación lineal en parcelas de 50 ha, según cultivo. 


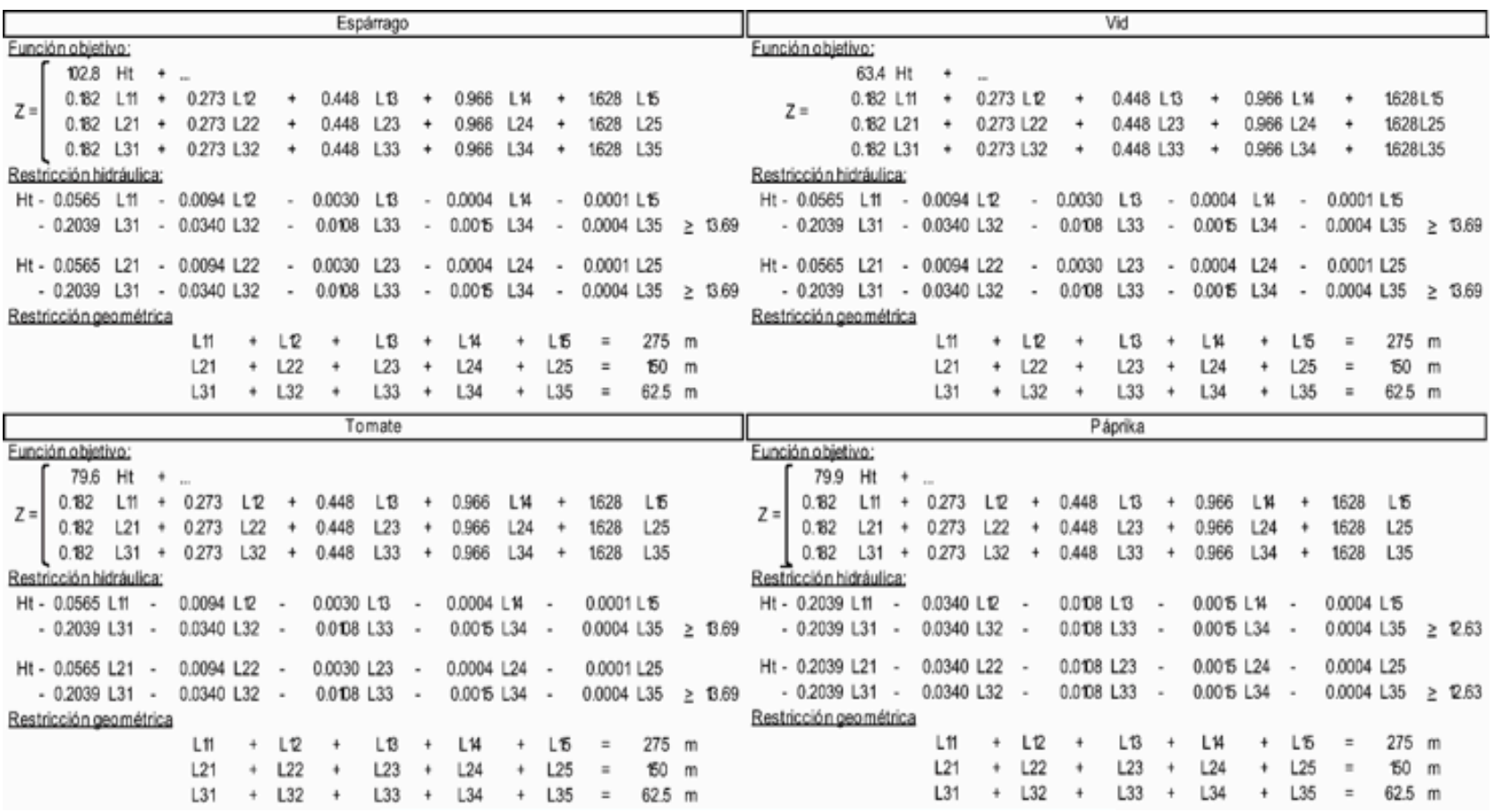

Figura 11. Modelos de programación lineal en parcelas de 20 ha, según cultivo.

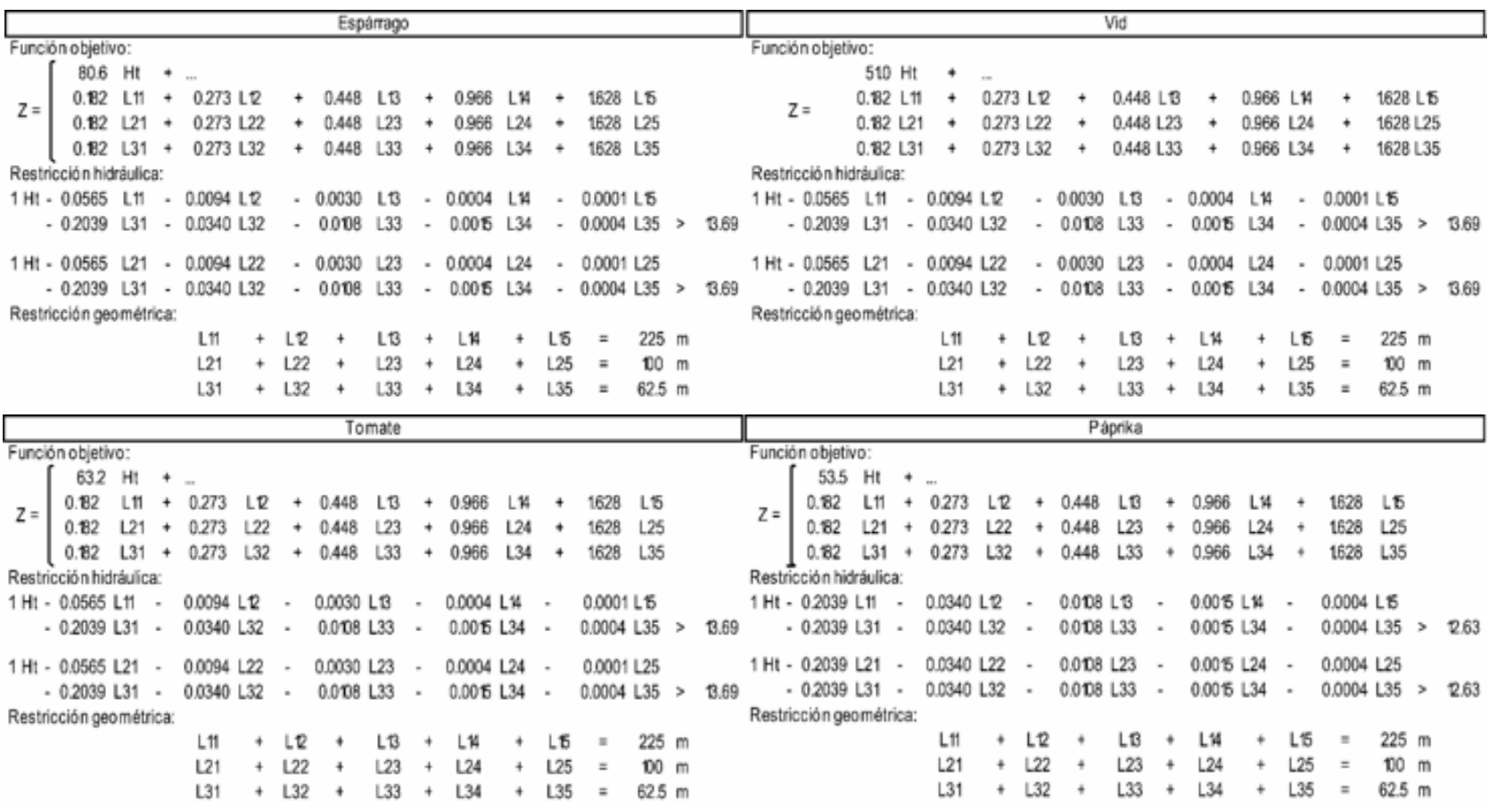

Figura 12. Modelos de programación lineal en parcelas de 15 ha, según cultivo.

El modelo para cebolla con cinco ha es: $\mathrm{Z}=40,3 \mathrm{Ht}+$ $0,182 \mathrm{~L} 11+0,273 \mathrm{~L} 12+0,448 \mathrm{~L} 13+0,966 \mathrm{~L} 14+1,628 \mathrm{~L} 15$; Ht- 0,2039L11- 0,0340L12- 0,0108L13- 0,0015L14$0,0004 \mathrm{~L} 15 \geq 12,63 ; \mathrm{L} 11+\mathrm{L} 12+\mathrm{L} 13+\mathrm{L} 14+\mathrm{L} 15=113$. En la Fig. 14 se detalla para el resto de cultivos.
Los 25 modelos de optimización se resolvieron con la opción "solver" del Excel. En la Tabla 7 se detallan las soluciones a los modelos de programación lineal formulados. 


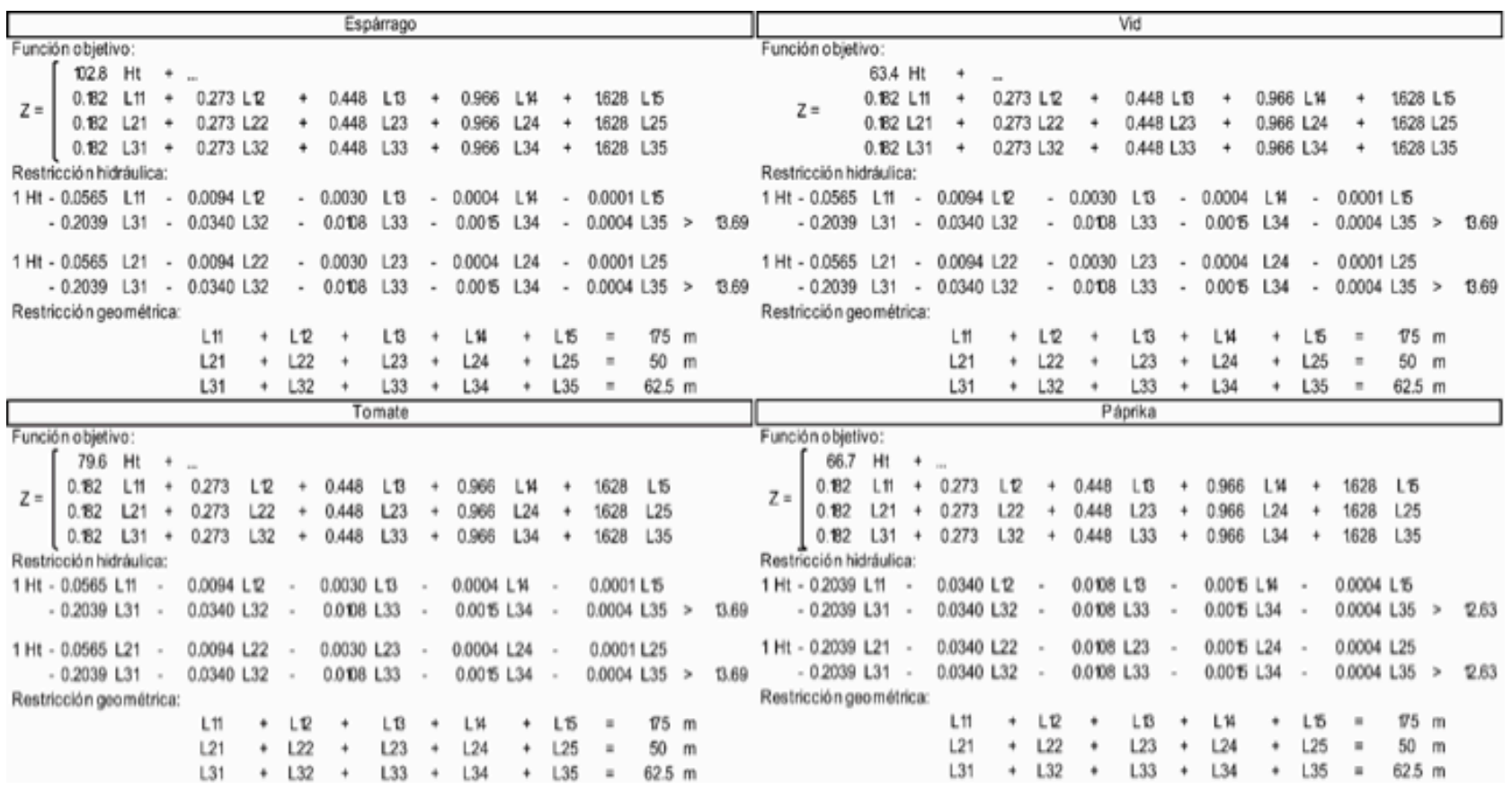

Figura 12. Modelos de programación lineal en parcelas de 10 ha, según cultivo.

Tabla 7. Solución a modelos de programación lineal: diámetros y longitudes de tubería

\begin{tabular}{|c|c|c|c|c|c|c|c|c|c|c|c|}
\hline \multicolumn{6}{|c|}{ Espárrago } & \multicolumn{6}{|c|}{ Vid } \\
\hline & 50 & 20 & 15 & 10 & 5 & & 50 & 20 & 15 & 10 & 5 \\
\hline $\mathrm{Ht}$ & 14,74 & 14,62 & 14,47 & 14,71 & 14,03 & $\mathrm{Ht}$ & 16,98 & 14,62 & 15.31 & 14,40 & 14,75 \\
\hline Tramo1 & $475 \mathrm{~m} 6 "$ & $275 \mathrm{~m}$ de $4 "$ & $225 \mathrm{~m}$ de $4 "$ & $175 \mathrm{~m}$ de $4 "$ & \multirow{2}{*}{$112,5 \mathrm{~m}$ de $4 "$} & \multirow{2}{*}{ Tramo1 } & $205 \mathrm{~m}$ de $4 "$ & \multirow{2}{*}{$275 \mathrm{~m}$ de $4 "$} & $40 \mathrm{~m}$ de $3 "$ & \multirow{2}{*}{$175 \mathrm{~m}$ de $4 "$} & \multirow{2}{*}{$112,5 \mathrm{~m}$ de $3 "$} \\
\hline \multirow{2}{*}{ Tramo2 } & $20 \mathrm{~m}$ de $4 "$ & $60 \mathrm{~m}$ de $3 "$ & $60 \mathrm{~m}$ de $3 "$ & \multirow{2}{*}{$50 \mathrm{~m}$ de $3 "$} & & & $270 \mathrm{~m}$ de $6 "$ & & $185 \mathrm{~m}$ de $4 "$ & & \\
\hline & $330 \mathrm{~m} \mathrm{6"}$ & 90m de 4" & 40m de 4" & & & \multirow{2}{*}{ Tramo2 } & $225 \mathrm{~m}$ de $4 "$ & $60 \mathrm{~m}$ de $3 "$ & \multirow{2}{*}{100 de $3 "$} & \multirow{2}{*}{$50 \mathrm{~m}$ de $3 "$} & \\
\hline \multirow{3}{*}{ Tramo3 } & 40m de 6" & \multirow{3}{*}{$62,5 \mathrm{~m}$ de $6 "$} & \multirow{3}{*}{$62,5 \mathrm{~m}$ de $6 "$} & \multirow{3}{*}{$62,5 \mathrm{~m}$ de $4 "$} & & & $125 \mathrm{~m}$ de $6 "$ & $90 \mathrm{~m}$ de $4 "$ & & & \\
\hline & $85 \mathrm{~m}$ de $8 "$ & & & & & Tramo3 & $125 \mathrm{~m}$ de $6 "$ & \multirow[t]{2}{*}{$62,5 \mathrm{~m}$ de $6 "$} & \multirow[t]{2}{*}{$62,5 \mathrm{~m} \mathrm{de} 4 "$} & \multirow[t]{2}{*}{$62,5 \mathrm{~m}$ de $4 "$} & \\
\hline & 350m 4" & & & & & Tramo4 & $350 \mathrm{~m}$ de 3 " & & & & \\
\hline \multicolumn{6}{|c|}{ Tomate } & \multicolumn{6}{|c|}{ Ají Páprika } \\
\hline & 50 & 20 & 15 & 10 & 5 & & 50 & 20 & 15 & 10 & 5 \\
\hline $\mathrm{Ht}$ & 15,07 & 14,62 & 14,46 & 14,40 & 14,03 & $\mathrm{Ht}$ & 15,86 & 14,34 & 15,78 & 15,20 & 13,85 \\
\hline \multirow{2}{*}{ Tramo1 } & \multirow{2}{*}{$475 \mathrm{~m} \mathrm{6"}$} & \multirow{2}{*}{$275 \mathrm{~m}$ de $4 "$} & \multirow{2}{*}{$225 \mathrm{~m}$ de $4 "$} & \multirow{2}{*}{$175 \mathrm{~m}$ de $4 "$} & \multirow{2}{*}{$112,5 \mathrm{~m}$ de $4 "$} & \multirow{2}{*}{ Tramo1 } & \multirow{2}{*}{$475 \mathrm{~m}$ de $6 "$} & $130 \mathrm{~m}$ de $4 "$ & $225 \mathrm{~m}$ de 4 " & $175 \mathrm{~m}$ de $4 "$ & $125 \mathrm{~m}$ de 4 " \\
\hline & & & & & & & & $145 \mathrm{~m}$ de $6 "$ & 225ाiा de 4 & 1/ Jill de 4 & 112, ind de 4 \\
\hline Tramo2 & $20 \mathrm{~m}$ de $4 "$ & $60 \mathrm{~m}$ de $3 "$ & $60 \mathrm{~m}$ de $3 "$ & $50 \mathrm{~m} \mathrm{de} 3 "$ & & Tramo2 & $20 \mathrm{~m}$ de $4 "$ & $150 \mathrm{~m}$ de $4 "$ & $60 \mathrm{~m}$ de $3 "$ & $50 \mathrm{~m}$ de $3 "$ & \\
\hline Hantive & $330 \mathrm{~m} 6 "$ & $90 \mathrm{~m}$ de $4 "$ & $40 \mathrm{~m}$ de $4 "$ & (1) & & Hanive & $330 \mathrm{~m}$ de $6 "$ & 150irit 4 & $40 \mathrm{~m} \mathrm{4"}$ & Sontuc & \\
\hline Tramo3 & $125 \mathrm{~m} 6 "$ & $62,5 \mathrm{~m}$ de $6 "$ & $62,5 \mathrm{~m}$ de $6 "$ & $62,5 \mathrm{~m}$ de $4 "$ & & Tramo3 & $125 \mathrm{~m}$ de $6 "$ & $62,5 \mathrm{~m}$ de $6 "$ & $62,5 \mathrm{~m}$ de 4 " & $62,5 \mathrm{~m}$ de $4 "$ & \\
\hline Tromp & $50 \mathrm{~m}$ de $3 "$ & & & & & Tremet 4 & $40 \mathrm{~m}$ de $4 "$ & & & & \\
\hline 1 ramo4 & $300 \mathrm{~m} \mathrm{4"}$ & & & & & 1 Iramo4 & $310 \mathrm{~m}$ de $6 "$ & & & & \\
\hline & & & Cebolla & & & & & & & & \\
\hline & 50 & 20 & 15 & 10 & 5 & & Ht: altura de & ombeo óptima & & & \\
\hline $\mathrm{Ht}$ & 14,70 & 13,13 & 13,80 & 15,20 & 13,85 & & & & & & \\
\hline Trampl $>$ & $300 \mathrm{~m} \mathrm{6"}$ & $275 \mathrm{~m}$ de $6=$ & $80 \mathrm{~m}$ de $4 "$ & $175 \mathrm{mde} 4$ & $1125 \mathrm{mde}$ & & & & & & \\
\hline Hantort & $175 \mathrm{~m} 8 "$ & 27 & $145 \mathrm{~m}$ de $6 "$ & 17 & $112,0110 \mathrm{~T}$ & & & & & & \\
\hline & & $20 \mathrm{~m}$ de $4 "$ & & & & & & & & & \\
\hline Tramo2 & $350 \mathrm{~m} 6$ & $130 \mathrm{~m}$ de $6 "$ & 100 de 4 & $50 \mathrm{~m} \mathrm{de} 3 "$ & & & & & & & \\
\hline Tramo3 & $125 \mathrm{~m} 8^{\prime \prime}$ & $62,5 \mathrm{~m}$ de $6 "$ & $62,5 \mathrm{~m}$ de $6 "$ & $62,5 \mathrm{~m}$ de $4 "$ & & & & & & & \\
\hline Tramo4 4 & $10 \mathrm{~m} \mathrm{4"}$ & & & & & & & & & & \\
\hline IICIIIT) & $340 \mathrm{~m} \mathrm{6"}$ & & & & & & & & & & \\
\hline
\end{tabular}


El sistema de bombeo está compuesto de una bomba sumergible con motor diesel que extrae agua del subsuelo y alimenta directamente el sistema de riego por medio del cabezal de riego, que se diseñó en función del caudal y presión de operación del sistema.

La presión requerida a la salida de la estación de bombeo (H) es: $\mathrm{H}=\mathrm{Ht}_{\text {optima }}+\mathrm{H}_{\text {succión del pozo }}+$ pérdida en el cabezal y conexiones de subunidad de riego; $\mathrm{H}=\mathrm{Ht}_{\text {optima }}+25 \mathrm{~m}+$ $10 \mathrm{~m}$. Donde: $\mathrm{H}_{\text {succión }}$ para explotación del agua subterránea es de 25 m (Baldeón, 2003).

\section{Evaluación económica}

Se realizó para un horizonte del proyecto de 10 años, con una tasa de interés efectiva de la banca comercial del $17 \%$ (i), evaluándose indicadores de rentabilidad como VANE, TIRE y B/C que se muestran en la Tabla 8.

Los costos de operación y mantenimiento se detallan en la Tabla 9 y los costos de cultivo en la Tabla 10. Detalles del metrado y costos del equipo de riego se muestran en la Tabla 11 .

En la Fig. 15 se visualiza la rentabilidad del proyecto para una tasa interna de retorno económica (TIRE) del $17 \%$ y se observa las parcelas que no son rentables al tener un TIRE menor al $17 \%$. Las parcelas de espárrago son rentables con tamaño de parcelas iguales o mayores a 20 ha, con un TIRE de 18,1\%, VANE de 9 mil dólares y una relación de $\mathrm{B} / \mathrm{C}$ de 1.1. En el caso de la vid son rentables con tamaño de parcela de 50 ha, con un TIRE de 17,4\%, VANE de 12 mil dólares y una relación $\mathrm{B} / \mathrm{C}$ de 1,0.

En el caso del tomate, cebolla y ají páprika, son rentables con todos los tamaños de parcela; siendo los indicadores de rentabilidad para 5 ha de tomate: $29 \%, 25$ mil dólares y 1,4 de TIRE, VANE y B/C, respectivamente. Para 5 ha de cebolla los indicadores son: $33 \%, 38$ mil dólares y 1,5 de TIRE, VANE y B/C, respectivamente. En el caso de 5 ha de páprika, los indicadores son $24 \%, 15$ mil dólares y 1,2 de TIRE, VANE y B/C, respectivamente.

\section{Conclusiones}

Los resultados de los 25 modelos de programación lineal y evaluación económica indican que los tamaños de parcela mínimos óptimos para el proyecto son de 20 ha para espárrago, 50 ha para vid y 5 ha para tomate, cebolla y ají páprika.

De los cinco cultivos propuestos, es la cebolla la que presenta la mayor rentabilidad seguida, en orden decreciente, del tomate, ají páprika, espárrago y vid. Son

Tabla 8. Indicadores de rentabilidad: VANE, TIRE y B/C

\begin{tabular}{|c|c|c|c|c|c|c|c|c|c|c|c|c|c|c|c|}
\hline \multirow{2}{*}{$\mathrm{Ha}$} & \multicolumn{3}{|c|}{ Espárrago } & \multicolumn{3}{|c|}{ Vid } & \multicolumn{3}{|c|}{ Tomate } & \multicolumn{3}{|c|}{ Cebolla } & \multicolumn{3}{|c|}{ Páprika } \\
\hline & VANE* & $\mathrm{B} / \mathrm{C}$ & TIRE & VANE* & $\mathrm{B} / \mathrm{C}$ & TIRE & VANE* & $\mathrm{B} / \mathrm{C}$ & TIRE & VANE* & $\mathrm{B} / \mathrm{C}$ & TIRE & VANE* & $\mathrm{B} / \mathrm{C}$ & TIRE \\
\hline 5 & -19 & 0,7 & 10,3 & -23 & 0,6 & 10,5 & 25 & 1,4 & 29,0 & 38 & 1,5 & 33,1 & 15 & 1,2 & 23,8 \\
\hline 10 & -8 & 09 & 15,2 & -17 & 0,8 & 14,2 & 79 & 1,8 & 41,9 & 110 & 2,1 & 49,9 & 66 & 1,7 & 36,7 \\
\hline 15 & -6 & 10 & 16,2 & -15 & 0,9 & 15,3 & 126 & 1,9 & 449 & 171 & 2,2 & 51,5 & 106 & 1,8 & 39,2 \\
\hline 20 & 9 & 11 & 181 & -8 & 1,0 & 16,3 & 185 & 2,1 & 51,1 & 235 & 2,2 & 53,8 & 154 & 1,9 & 432 \\
\hline 50 & 39 & 1,1 & 18,9 & 12 & 1,0 & 17,4 & 482 & 2,2 & 54,7 & 629 & 2,5 & 60,7 & 410 & 2,0 & 46,8 \\
\hline
\end{tabular}

* en miles de dólares.

Tabla 9. Costos de la operación y mantenimiento del sistema de riego

\begin{tabular}{lrrrrrrrrrr}
\hline Cultivo & \multicolumn{4}{c}{ Costo anual de energía (\$) } & \multicolumn{5}{c}{ Costo (oper+manten) } \\
& 50 ha & 20 ha & 15 ha & 10 ha & 5 ha & 50 ha & 20 ha & 15 ha & 10 ha & 5 ha \\
\hline Espárrago & 11557 & 4623 & 3467 & 2311 & 1156 & 17336 & 6934 & 5201 & 3467 & 1734 \\
Vid & 6424 & 2569 & 1927 & 1285 & 642 & 9636 & 3854 & 2891 & 1927 & 964 \\
Tomate & 8532 & 3413 & 2560 & 1706 & 853 & 12798 & 5119 & 3839 & 2560 & 1280 \\
Ají Páprika & 6855 & 2742 & 2056 & 1371 & 685 & 10282 & 4113 & 3085 & 2056 & 1028 \\
Cebolla & 10170 & 4068 & 3051 & 2034 & 1017 & 15255 & 6102 & 4577 & 3051 & 1526 \\
\hline
\end{tabular}

Tabla 10. Costos de producción, rendimiento y precio de los cultivos

\begin{tabular}{|c|c|c|c|c|c|c|c|}
\hline \multirow{2}{*}{ Detalle (año 2009) } & \multicolumn{2}{|c|}{ Espárrago UC 157 F1 } & \multicolumn{2}{|c|}{ Vid Quebranta } & \multirow{2}{*}{$\begin{array}{c}\text { Tomate } \\
\text { RíoGrande }\end{array}$} & \multirow{2}{*}{$\begin{array}{l}\text { Cebolla } \\
\text { Amarilla }\end{array}$} & \multirow{2}{*}{$\begin{array}{c}\text { Aji } \\
\text { Páprika }\end{array}$} \\
\hline & instalación & mantenimiento & instalación & mantenimiento & & & \\
\hline $\begin{array}{l}\text { Costo de producción } \\
\text { \$/ha }\end{array}$ & 5000 & 4000 & 10000 & 2500 & 4000 & 3000 & 3000 \\
\hline Rendimiento kg/ha & 3000 & 10000 & 8000 & 18000 & 70000 & 4200 & 5000 \\
\hline Precio $\$ / \mathrm{kg}$ & 0,75 & 0,75 & 0,39 & 0,39 & 0,11 & 0,18 & 1,30 \\
\hline
\end{tabular}


Tabla 11. Metrado y costos del equipo de riego en parcelas según cultivo

\begin{tabular}{|c|c|c|c|c|c|c|c|c|c|c|c|c|c|c|}
\hline \multirow[b]{2}{*}{ Detalle } & \multirow[b]{2}{*}{ Unidad } & \multirow[b]{2}{*}{$\begin{array}{c}\text { C.U. } \\
\text { Espárrago }\end{array}$} & \multicolumn{5}{|c|}{50 ha ( 80 subunidades de riego) } & \multicolumn{3}{|c|}{20 ha (32 subunidades) } & \multicolumn{4}{|c|}{15 ha (24 subunidades) } \\
\hline & & & Vid & Tomate & Páprika & Cebolla & $\begin{array}{l}\text { Esparra, } \\
\text { Vid, } \\
\text { Tomate }\end{array}$ & Páprika & Cebolla & $\begin{array}{l}\text { Espárrago } \\
\text { Tomate }\end{array}$ & Vid & Páprika & Cebolla & \\
\hline Subunidad de Riego & & & 209137 & 209137 & 209137 & 220253 & 220253 & 83855 & 88493 & 88493 & 63620 & 63620 & 66859 & 66859 \\
\hline \multicolumn{15}{|l|}{ En manguera de Goteo: } \\
\hline $\begin{array}{l}\text { PEBD } ø=16 \mathrm{~mm} \text { con } \\
3 \mathrm{got} / \mathrm{mt}\end{array}$ & $\mathrm{m}$ & 0,65 & 156000 & 156000 & 156000 & 156000 & 156000 & 62400 & 62400 & 62400 & 46800 & 46800 & 46800 & 46800 \\
\hline $\begin{array}{l}\text { Anillo conector } \\
\text { rápido de } \mathrm{PE}\end{array}$ & unid. & 0,40 & 1920 & 1920 & 1920 & 1920 & 1920 & 768 & 768 & 768 & 576 & 576 & 576 & 576 \\
\hline $\begin{array}{l}\text { Tapón final PE } \\
ø=16 \mathrm{~mm}\end{array}$ & unid. & 0,10 & 480 & 480 & 480 & 480 & 480 & 192 & 192 & 192 & 144 & 144 & 144 & 144 \\
\hline \multicolumn{15}{|l|}{ En el manifold: } \\
\hline Tubería PVC ø=2" & $\mathrm{m}$ & 1,38 & 6624 & 6624 & 6624 & 0 & 0 & 2650 & 0 & 0 & 1987 & 1987 & 0 & 0 \\
\hline Tubería PVC ø=3" & $\mathrm{m}$ & 2,08 & 0 & 0 & 0 & 9984 & 9984 & 0 & 3994 & 3994 & 0 & 0 & 2995 & 2995 \\
\hline Tapón final PVC & unid. & 0,40 & 32 & 32 & 32 & 32 & 32 & 13 & 13 & 13 & 10 & 10 & 10 & 10 \\
\hline $\begin{array}{l}\text { Montura 2"-3/4", } \\
\mathrm{L}=1.1 \mathrm{~m}\end{array}$ & unid. & 8,00 & 6400 & 6400 & 6400 & 0 & 0 & 2560 & 0 & 0 & 1920 & 1920 & 0 & 0 \\
\hline $\begin{array}{l}\text { Montura 3"-3/4", } \\
\mathrm{L}=1.1 \mathrm{~m}\end{array}$ & unid. & 12,00 & 0 & 0 & 0 & 9600 & 9600 & 0 & 3840 & 3840 & 0 & 0 & 2880 & 2880 \\
\hline $\begin{array}{l}\text { Válvula de acople } \\
\text { rápido de } 3 / 4 "\end{array}$ & unid. & 15,00 & 12000 & 12000 & 12000 & 12000 & 12000 & 4800 & 4800 & 4800 & 3600 & 3600 & 3600 & 3600 \\
\hline $\begin{array}{l}\text { Conector PEAD con } \\
6 \text { salidas }\end{array}$ & unid. & 1.55 & 1240 & 1240 & 1240 & 1240 & 1240 & 496 & 496 & 496 & 372 & 372 & 372 & 372 \\
\hline \multicolumn{15}{|l|}{ En el arco: } \\
\hline $\begin{array}{l}\text { Tubería PVC negro } \\
\varnothing=2 "\end{array}$ & $\mathrm{~m}$ & 2,00 & 480 & 480 & 480 & 0 & 0 & 192 & 0 & 0 & 144 & 144 & 0 & 0 \\
\hline $\begin{array}{l}\text { Tubería PVC negro } \\
\varnothing=3 "\end{array}$ & $\mathrm{~m}$ & 3,20 & 0 & 0 & 0 & 768 & 768 & 0 & 307 & 307 & 0 & 0 & 230 & 230 \\
\hline Codo $90^{\circ}$ de PVC 2" & unid. & 2,45 & 392 & 392 & 392 & 0 & 0 & 157 & 0 & 0 & 118 & 118 & 0 & 0 \\
\hline Codo $90^{\circ}$ de PVC $3 "$ & $\mathrm{~m}$ & 4,90 & 0 & 0 & 0 & 784 & 784 & 0 & 314 & 314 & 0 & 0 & 235 & 235 \\
\hline Niple 2", L=1.10m & unid. & 2,70 & 216 & 216 & 216 & 0 & 0 & 86 & 0 & 0 & 65 & 65 & 0 & 0 \\
\hline Niple 3", $\mathrm{L}=1.10 \mathrm{~m}$ & unid. & 4,10 & 0 & 0 & 0 & 328 & 328 & 0 & 131 & 131 & 0 & 0 & 98 & 98 \\
\hline $\begin{array}{l}\text { Válvula acople } \\
\text { rápido de } 3 / 4 "\end{array}$ & unid. & 15,00 & 2400 & 2400 & 2400 & 2400 & 2400 & 960 & 960 & 960 & 720 & 720 & 720 & 720 \\
\hline $\begin{array}{l}\text { Regulador de } \\
\text { presión de 2" }\end{array}$ & unid. & 150 & 12000 & 12000 & 12000 & 0 & 0 & 4800 & 0 & 0 & 3600 & 3600 & 0 & 0 \\
\hline $\begin{array}{l}\text { Regulador de } \\
\text { presión de 3" }\end{array}$ & unid. & 220 & 0 & 0 & 0 & 17600 & 17600 & 0 & 7040 & 7040 & 0 & 0 & 5280 & 5280 \\
\hline $\begin{array}{l}\text { Válvula de paso } \\
\text { de 2" }\end{array}$ & unid. & 27,00 & 2160 & 2160 & 2160 & 0 & 0 & 864 & 0 & 0 & 648 & 648 & 0 & 0 \\
\hline $\begin{array}{l}\text { Válvula de paso } \\
\text { de 3" }\end{array}$ & unid. & 40,00 & 0 & 0 & 0 & 3200 & 3200 & 0 & 1280 & 1280 & 0 & 0 & 960 & 960 \\
\hline $\begin{array}{l}\text { Inyector fertilizante } \\
\text { tipo venturi }\end{array}$ & unid. & 1000 & 2000 & 2000 & 2000 & 2000 & 2000 & 1000 & 1000 & 1000 & 1000 & 1000 & 1000 & 1000 \\
\hline \multicolumn{15}{|c|}{ Arco, solo para lavado de sales: } \\
\hline $\begin{array}{l}\text { Aspersor } \\
\mathrm{Q}=1.44 \mathrm{~m} 3 / \mathrm{h} \\
(12 \times 12)\end{array}$ & $\mathrm{m}$ & 10,68 & 1068 & 1068 & 1068 & 427 & 427 & 427 & 214 & 214 & 427 & 427 & 214 & 214 \\
\hline Elevador $\mathrm{h}=1.0 \mathrm{~m}$ & unid. & 0,95 & 95 & 95 & 95 & 38 & 38 & 38 & 19 & 19 & 38 & 38 & 19 & 19 \\
\hline $\begin{array}{l}\text { Manguera PEMD, } \\
\emptyset=25 \mathrm{~mm}\end{array}$ & unid. & 0,72 & 3600 & 3600 & 3600 & 1440 & 1440 & 1440 & 720 & 720 & 1440 & 1440 & 720 & 720 \\
\hline Tapón 25mm & unid. & 0,30 & 30 & 30 & 30 & 12 & 12 & 12 & 6 & 6 & 12 & 12 & 6 & 6 \\
\hline Línea de Distribución & & & 45464 & 29462 & 40605 & 48481 & 53309 & 7571 & 10989 & 19131 & 7737 & 4462 & 7737 & 9182 \\
\hline Tubería PVC 3" & $\mathrm{m}$ & 2,08 & 0 & 1456 & 416 & 0 & 0 & 374 & 0 & 0 & 499 & 1165 & 499 & 0 \\
\hline Tubería PVC 4" & $\mathrm{m}$ & 3,41 & 2660 & 5865 & 2319 & 546 & 68 & 4126 & 3819 & 273 & 4126 & 2097 & 4126 & 2455 \\
\hline Tubería PVC 6" & $\mathrm{m}$ & 7,35 & 22418 & 11613 & 21830 & 28224 & 24108 & 919 & 3418 & 10988 & 919 & 0 & 919 & 3344 \\
\hline Tubería PVC 8" & $\mathrm{m}$ & 12,38 & 2105 & 0 & 0 & 0 & 6438 & & & & & & & \\
\hline $\begin{array}{l}\text { Unión doble } \\
\text { campana 3" }\end{array}$ & unid. & 3,50 & 0 & 490 & 140 & 0 & 0 & 126 & 0 & 0 & 168 & 392 & 168 & 0 \\
\hline $\begin{array}{l}\text { Unión doble } \\
\text { campana 4" }\end{array}$ & unid. & 5,00 & 780 & 1720 & 680 & 160 & 20 & 1210 & 1120 & 80 & 1210 & 615 & 1210 & 720 \\
\hline $\begin{array}{l}\text { Unión doble } \\
\text { campana 6" }\end{array}$ & unid. & 25,00 & 15250 & 7900 & 14850 & 19200 & 16400 & 625 & 2325 & 7475 & 625 & 0 & 625 & 2275 \\
\hline $\begin{array}{l}\text { Unión doble } \\
\text { campana } 8 "\end{array}$ & unid. & 52,00 & 1768 & 0 & 0 & 0 & 5408 & & & & & & & \\
\hline
\end{tabular}




\begin{tabular}{|c|c|c|c|c|c|c|c|c|c|c|c|c|c|c|}
\hline $\begin{array}{l}\text { Reducción PVC } \\
\text { 8"-6" }\end{array}$ & unid. & 44,50 & 89 & 0 & 0 & 0 & 356 & & & & & & & \\
\hline $\begin{array}{l}\text { Reducción PVC } \\
8 \text { "-4" }\end{array}$ & unid. & 40,50 & 81 & 0 & 0 & 0 & 0 & & & & & & & \\
\hline $\begin{array}{l}\text { Reducción PVC } \\
6 "-4 "\end{array}$ & unid. & 18,30 & 73 & 146 & 110 & 110 & 37 & & & & & & & \\
\hline $\begin{array}{l}\text { Reducción PVC } \\
6 \text { "-3" }\end{array}$ & unid. & 15,20 & 0 & 30 & 0 & 0 & 0 & & & & & & & \\
\hline $\begin{array}{l}\text { Reducción PVC } \\
4 "-3 "\end{array}$ & unid. & 9,72 & 0 & 0 & 19 & 0 & 0 & 39 & 0 & 0 & 39 & 78 & 39 & 0 \\
\hline $\begin{array}{l}\text { Reducción PVC } \\
6 "-4 "\end{array}$ & & & & & & & & 37 & 146 & 73 & 37 & 0 & 37 & 146 \\
\hline Tee 4" & & & & & & & & 115 & 0 & 0 & 115 & 115 & 115 & 0 \\
\hline Tee 6" & unid. & 40,23 & 241 & 241 & 241 & 241 & 0 & 0 & 161 & 241 & 0 & 0 & 0 & 241 \\
\hline Tee 8" & unid & 79,15 & 0 & 0 & 0 & 0 & 475 & & & & & & & \\
\hline Unidad de Bombeo & & & 29264 & 29264 & 29264 & 25514 & 25514 & 13657 & 13657 & 13657 & 13657 & 13657 & 13657 & 13657 \\
\hline Hidrociclón 4" & unid. & 400 & 2000 & 2000 & 2000 & 1600 & 1600 & 800 & 800 & 800 & 800 & 800 & 800 & 800 \\
\hline Filtro de malla 4" & unid. & 500 & 2500 & 2500 & 2500 & 2000 & 2000 & 1000 & 1000 & 1000 & 1000 & 1000 & 1000 & 1000 \\
\hline Tubería de aire de 2" & unid. & 100 & 500 & 500 & 500 & 400 & 400 & 200 & 200 & 200 & 200 & 200 & 200 & 200 \\
\hline $\begin{array}{l}\text { Válvula volumétrica } \\
8 \text { " }\end{array}$ & unid. & 3900 & 3900 & 3900 & 3900 & 3900 & 3900 & & & & & & & \\
\hline $\begin{array}{l}\text { Válvula volumétrica } \\
6 "\end{array}$ & & & & & & & & 2800 & 2800 & 2800 & 2800 & 2800 & 2800 & 2800 \\
\hline Manómetro & unid. & 28,50 & 114 & 114 & 114 & 114 & 114 & 57 & 57 & 57 & 57 & 57 & 57 & 57 \\
\hline $\begin{array}{l}\text { Control para } \\
\text { manómetro }\end{array}$ & unid. & 6,82 & 68 & 68 & 68 & 55 & 55 & 27 & 27 & 27 & 27 & 27 & 27 & 27 \\
\hline $\begin{array}{l}\text { Válvula de control } \\
4 "\end{array}$ & unid. & 68,20 & 682 & 682 & 682 & 546 & 546 & 273 & 273 & 273 & 273 & 273 & 273 & 273 \\
\hline 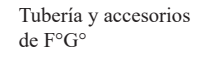 & unid. & 1000 & 1000 & 1000 & 1000 & 900 & 900 & 500 & 500 & 500 & 500 & 500 & 500 & 500 \\
\hline \multicolumn{15}{|l|}{$\begin{array}{l}\text { Bomba sumerg } \\
10 \mathrm{HP}+\text { motor }\end{array}$} \\
\hline $\begin{array}{l}\text { Bomba sumerg } \\
20 \mathrm{HP}+\text { motor }\end{array}$ & & & & & & & & 8000 & 8000 & 8000 & 8000 & 8000 & 8000 & 8000 \\
\hline $\begin{array}{l}\text { Bomba sumerg } \\
50 \mathrm{HP}+\text { motor }\end{array}$ & unid. & 18500 & 18500 & 18500 & 18500 & 0 & 0 & & & & & & & \\
\hline $\begin{array}{l}\text { Bomba sumerg } \\
40 \mathrm{HP}+\text { motor }\end{array}$ & unid. & 16000 & 0 & 0 & 0 & 16000 & 16000 & & & & & & & \\
\hline \multicolumn{3}{|l|}{ Equipo de Riego } & 283866 & 267864 & 279006 & 294248 & 299077 & 105082 & 113139 & 121281 & 85015 & 81739 & 88254 & 89699 \\
\hline \multicolumn{3}{|c|}{ Instalación del Equipo de Riego (5\%) } & 14193 & 13393 & 13950 & 14712 & 14954 & 5254 & 5657 & 6064 & 4251 & 4087 & 4413 & 4485 \\
\hline \multicolumn{3}{|c|}{ Perforación y desarrollo del pozo (prof=25m) } & 25000 & 25000 & 25000 & 25000 & 25000 & 25000 & 25000 & 25000 & 25000 & 25000 & 25000 & 25000 \\
\hline COSTO TO & AL año 2009 & U.S \$ & 323059 & 306257 & 317956 & 333961 & 339030 & 135337 & 143796 & 152345 & 114266 & 110826 & 117667 & 119184 \\
\hline
\end{tabular}

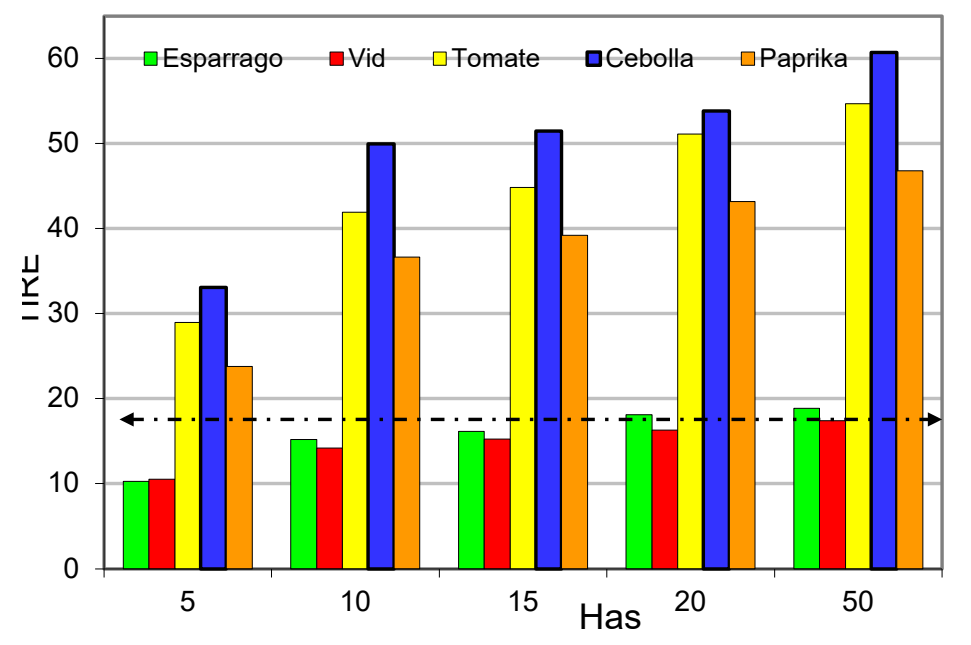

Figura 15. Tasa interna de retorno económico (TIRE) según tamaño de parcela y cultivo. 
los cultivos transitorios (tomate, cebolla y páprika) los que, en general, presentan mayor rentabilidad que los cultivos permanentes (espárrago y vid).

Acorde con el análisis de sensibilidad, para una reducción de un $20 \%$ en el rendimiento, se obtiene que la cebolla sigue siendo rentable en todos los tamaños de parcela; en cambio para el tomate y ají páprika solo resultó rentable en tamaños de parcela de 10, 15, 20 y 50 ha. En el caso de los cultivos permanentes (espárrago y vid), ninguno resultó rentable.

\section{Literatura citada}

Ascencios,TD. 1999. Diseño de la red de tuberías y costo de operación en los sistemas de riego a presión. Revista Anales Cientificos. Universidad Nacional Agraria La Molina, Lima, Perú, pp. 110-134.
Baldeón, QW. 2003. Metodología de autocalibración para el modelo de simulación del acuífero de Villacurí. En Maestría de Recursos Hídricos - Escuela de Postgrado. Lima, Perú: Universidad Nacional Agraria La Molina.

Bliesner, R.; Keller, J. 2001. Sprinkle and Trickle Irrigation. The Blackburn Press, p. 652.

Georgiadis, MC.; Schilling, G.; Rotstein, GE. y Macchietto.S.1999. Ageneral mathematical programming approach for process plant layout. Computers \& Chemical Engineering, 23: 823-840.

González-Cebollada, C.; Macarulla, B. 2012. Comparative analysis of design methods of pressurized irriation networks. Irrigation and Drainage, 61: 1-9 doi: 10.1002/ird.657.

Swamee, PK.; Sharma, AK. 2013. Optimal design of a sewer line using Linear Programming. Applied Mathematical Modelling, 37: 4430-4439. 